\title{
Nonlocal Mumford-Shah Regularizers for Color Image Restoration
}

\author{
Miyoun Jung, Xavier Bresson, Tony F. Chan, and Luminita A. Vese
}

\begin{abstract}
We introduce a class of color image restoration algorithms based on the Mumford-Shah model and nonlocal image information. The standard approximations of Ambrosio-Tortorelli and Shah models are defined to work in a small local neighborhood, which are sufficient to denoise smooth regions with sharp boundaries. However, textures are not local in nature and require semi-local/non-local information to be denoised efficiently. Inspired from recent works (NL-means of Buades, Coll, Morel and NL-TV of Gilboa, Osher), we extend the local Ambrosio-Tortorelli and Shah approximations to Mumford-Shah functional to novel nonlocal formulations, for better restoration of fine structures and textures. We present several applications of the proposed nonlocal MS regularizers in image processing such as color image denoising, color image deblurring in the presence of Gaussian or impulse noise, color image inpainting, color image super-resolution, and color filter array demosaicing. In all the applications, the proposed nonlocal regularizers produce superior results over the local ones, especially in image inpainting with large missing regions. We also prove several characterizations of minimizers based on dual norm formulations.
\end{abstract}

\section{Index Terms}

Mumford-Shah regularizer, non-local method, impulse noise, denoising, deblurring, inpainting, super-resolution, demosaicing.

\section{INTRODUCTION}

We consider the degradation model of a color image formalized as

$$
f=H u+n, \quad\left(f^{i}=H^{i} u^{i}+n^{i}, i=r, g, b\right)
$$

where $H$ is a linear operator accounting for some blurring, sub-sampling, or missing pixels (so that the observed data $f$ loses some portion of the original image $u$ we wish to recover), and $n$ is an additive noise. The problem (1) is a highly ill-posed problem, thus we formulate the restoration problem within the variational framework as: $\inf _{u}\{\Phi(f-H u)+\Psi(\nabla u)\}$, where $\Phi$ defines a data-fidelity term, and $\Psi$ defines the regularization that enforces a smoothness constraint on $u$, depending on its gradient $\nabla u$.

The regularization term $\Psi$ alleviates the ill-posedness of the inverse problem by reflecting some a priori properties. Several edge-preserving regularization terms were suggested in the literature, including [38], [66], [65], [6], [67], [14], [9]. These traditional regularization terms are based on local image operators, which denoise and preserve edges and smooth regions very well, but may not deal well with fine structures like texture during the restoration process because textures are not local in nature.

Recently, new image denoising models have been developed, based on non-local image operators, to better deal with textures. Buades et al [20] introduced the nonlocal means filter, producing excellent denoising results as well as being used for demosaicing [22]. Kindermann et al [49], and Gilboa-Osher [40], [41] formulated a variational framework of NL-means by proposing nonlocal regularizing functionals. Lou et al [50] used the nonlocal total variation of Gilboa-Osher (NL/TV) in grey-scale image deblurring in the presence of Gaussian noise. Moreover, Peyré et al [63] used NL/TV for grey-scale image inpainting, super-resolution of a single image, and compressive

This work has been supported by grants NSF-DMS 031222 and 0714945.

M. Jung, X. Bresson are with the Department of Mathematics, University of California, Los Angeles, CA 90095, USA (email: gomtaeng, xbresson@math.ucla.edu).

T.F. Chan is now in the Hong Kong university of Science and Technology, Hong Kong (email: chan@math.ucla.edu).

L.A. Vese is with the Department of Mathematics, University of California, Los Angeles, CA 90095, USA (corresponding author to provide phone: 310-825-4201; fax: 310-206-2679; email: lvese@ math.ucla.edu). 
sensing, and Zhang et al [77] also applied NL/TV in compressive sensing. Protter et al. [64] generalized the NL-means filter to super-resolution.

Previous work using nonlocal methods has been done for the Gaussian noise model, but no study has been developed for the impulse noise model using non-local information. However, the impulsive noise model was studied in the local case. Bar et al [9] used the Ambrosio-Tortorelli and Shah approximations to Mumford-Shah regularizing functional for color image deblurring in the presence of impulse noise, producing better restorations than total variation (TV) regularizer, and moreover providing the edge set detected concurrently with the restoration process. We propose in this paper nonlocal versions of Ambrosio-Tortorelli [6] and Shah [67] approximations to the Mumford-Shah regularizer for the multichannel case. We also propose (a) several applications of the NL/MS for color image denoising, deblurring in the presence of Gaussian or impulse noise, inpainting with large missing portion, super-resolution of a single image, color filter array demosaicing, and (b) an efficient preprocessing step to compute the weights $w$ in the deblurring-denoising model in the presence of impulse noise. In all the applications, we show that the proposed nonlocal regularizers produce superior results over the local ones. We mention that preliminary results of this work have been presented in conference proceedings [46], [47].

\section{BACKGROUND}

a) Local regularizers: We recall two approximations of the Mumford-Shah regularizing functional [58] that have been used in several segmentation and restoration algorithms. The MS regularizer, depending on the image $u: \Omega \rightarrow \mathbb{R}$ and on its edge set $K \subset \Omega$, giving preference to piecewise smooth images, is

$$
\Psi^{M S}(u, K)=\beta \int_{\Omega \backslash K}|\nabla u|^{2} d x+\alpha \int_{K} d \mathcal{H}^{1},
$$

where $|\nabla u|=\sqrt{u_{x_{1}}^{2}+u_{x_{2}}^{2}}$ with $x=\left(x_{1}, x_{2}\right)$, and $\mathcal{H}^{1}$ is the one-dimensional Hausdorff measure. The first term enforces smoothness of $u$ everywhere except on the edge set $K$, and the second one minimizes the total length of edges. It is difficult to minimize in practice the non-convex MS functional.

There are several numerical approaches for minimizing the MS regularizing functional, one being the phase field approach using $\Gamma$-convergence with applications to image deblurring and denoising [7], and image inpainting [68].

More specifically, Ambrosio and Tortorelli [6] approximated this functional by a sequence of regular functionals $\Psi_{\epsilon}$ using the $\Gamma$-Convergence. The edge set $K$ is represented by a smooth auxiliary function $v$. Thus we have an approximation to $\Psi^{M S}$ as [6]:

$$
\Psi_{\epsilon}^{M S H^{1}}(u, v)=\beta \int_{\Omega} v^{2}|\nabla u|^{2} d x+\alpha \int_{\Omega}\left(\epsilon|\nabla v|^{2}+\frac{(v-1)^{2}}{4 \epsilon}\right) d x,
$$

where $0 \leq v(x) \leq 1$ represents the edges: $v(x) \approx 0$ if $x \in K=J_{u}$ (the jump set of $u$ ), and $v(x) \approx 1$ otherwise, $\epsilon$ is a small positive constant, $\alpha, \beta$ are positive weights. After incorporating the data fidelity term, a minimizer $u=u_{\epsilon}$ of $\Psi_{\epsilon}^{M S H^{1}}$ approaches a minimizer $u$ of $\Psi^{M S}$ as $\epsilon \rightarrow 0$.

An alternative approach is the total variation [65] proposed by Rudin, Osher, and Fatemi, so called TV regularizer: given a locally integrable function $u$,

$$
\Psi^{T V}(u)=\int_{\Omega}|D u|=\sup \left\{\int_{\Omega} u \nabla \cdot \phi d x: \phi \in C_{c}^{1}(\Omega, \mathbb{R}),\|\phi\|_{L^{\infty}(\Omega)} \leq 1\right\} \approx \int_{\Omega}|\nabla u| d x .
$$

Because of its benefit of preserving edges (which have high gradient levels) and convexity, the total variation has been widely used in image restoration.

Shah [67] suggested a modified version of the Ambrosio-Tortorelli approximation to the MS functional by replacing the quadratic term $|\nabla u|^{2}$ by $|\nabla u|$ in the first term:

$$
\Psi_{\epsilon}^{M S T V}(u, v)=\beta \int_{\Omega} v^{2}|\nabla u| d x+\alpha \int_{\Omega}\left(\epsilon|\nabla v|^{2}+\frac{(v-1)^{2}}{4 \epsilon}\right) d x .
$$

This functional $\Gamma$-converges to the other functional $\Psi^{M S T V}[1]$ :

$$
\Psi^{M S T V}(u)=\beta \int_{\Omega \backslash K}|\nabla u| d x+\alpha \int_{K} \frac{\left|u^{+}-u^{-}\right|}{1+\left|u^{+}-u^{-}\right|} d \mathcal{H}^{1}+\left|D_{c} u\right|(\Omega),
$$


where $u^{+}$and $u^{-}$denote the image values on two sides of the jump set $K=J_{u}$ of $u$, and $D_{c} u$ is the Cantor part of the measure-valued derivative $D u$. Note that the non-convex term $\frac{\left|u^{+}-u^{-}\right|}{1+\left|u^{+}-u^{-}\right|}$is similar with the prior regularization by Geman-Reynolds [38]. We observe that this regularizing functional is similar to the total variation of $u \in B V(\Omega)$ that can be written, for $K=J_{u}$, as:

$$
\int_{\Omega}|D u|=\int_{\Omega \backslash K}|\nabla u| d x+\int_{K}\left|u^{+}-u^{-}\right| d \mathcal{H}^{1}+\left|D_{c} u\right|(\Omega) .
$$

By comparing the second terms, we see that MSTV regularizer does not penalize the jump part as much as TV regularizer does.

In the case of color images, Blomgren and Chan [14] presented a color TV regularization by coupling the channels

$$
\sqrt{\left[\int\left|\nabla u^{r}\right|\right]^{2}+\left[\int\left|\nabla u^{g}\right|\right]^{2}+\left[\int\left|\nabla u^{b}\right|\right]^{2}} .
$$

The natural generalization of TV regularization to color images with coupled channels takes the form [2]:

$$
\Psi^{T V}=\int_{\Omega}\|\nabla u\| d x=\int_{\Omega} \sqrt{\left|\nabla u^{r}\right|^{2}+\left|\nabla u^{g}\right|^{2}+\left|\nabla u^{b}\right|^{2}} d x,
$$

which was analyzed in [17]. Both channel coupling regularizers yield similar experimental results.

Bar et al [9] used the color versions of Mumford-Shah regularizers for color image deblurring-denoising, by replacing the scalar-valued version of $|\nabla u|$ by the vector-valued version $\|\nabla u\|$ defined above, such that

$$
\begin{aligned}
& \Psi_{\epsilon}^{M S H^{1}}(u, v)=\beta \int_{\Omega} v^{2}\|\nabla u\|^{2} d x+\alpha \int_{\Omega}\left(\epsilon|\nabla v|^{2}+\frac{(v-1)^{2}}{4 \epsilon}\right) d x, \\
& \Psi_{\epsilon}^{M S T V}(u, v)=\beta \int_{\Omega} v^{2}\|\nabla u\| d x+\alpha \int_{\Omega}\left(\epsilon|\nabla v|^{2}+\frac{(v-1)^{2}}{4 \epsilon}\right) d x .
\end{aligned}
$$

Note that, in both MS regularizers, the scalar-valued edge map $v$ is common for the three channels and provides the necessary coupling between color.

b) Nonlocal methods: Nonlocal methods in image processing have been explored in many papers because they are well adapted to texture denoising while the standard denoising models working with local image information seem to consider texture as noise, which results in losing textures. Nonlocal methods are generalized from the neighborhood filters (e.g. Yaroslavsky filter [76], bilateral filter [74], Susan filter [69]) and patch based methods [32], [75]. The idea of neighborhood filter is to restore a pixel by averaging the values of neighboring pixels with a similar grey level value.

Buades et al. [20] generalized this idea by applying the patch-based method, and proposed the well-known non-local means (or NL-means) filter for denoising, given by

$$
\begin{aligned}
N L u(x) & =\frac{1}{C(x)} \int_{\Omega} e^{-\frac{d_{a}(u(x), u(y))}{h^{2}}} u(y) d y \\
d_{a}(u(x), u(y)) & =\int_{R^{2}} G_{a}(t)\|u(x+t)-u(y+t)\|^{2} d t
\end{aligned}
$$

where $u(y)$ is the color at $\mathrm{y}, d_{a}$ is the patch distance, $G_{a}$ is the Gaussian kernel with standard deviation $a$ determining the patch size, $C(x)=\int_{\Omega} e^{-\frac{d_{a}(u(x), u(y))}{h^{2}}} d y$ is the normalization factor, and $h$ is the filtering parameter which corresponds to the noise level; usually we set it to be the standard deviation of the noise. The NL-means not only compares the grey level at a single point but the geometrical configuration in a whole neighborhood (patch). 
c) Nonlocal regularizers: Nonlocal filtering can be understood as a quadratic regularization based on a nonlocal graph, as detailed for instance in the geometric diffusion framework of Coifman et al. [28], which was applied to non-local image denoising by Szlam et al. [71]. Denoising using quadratic penalization on image graphs was studied by Gilboa and Osher for image restoration and segmentation [40]. These quadratic regularizations were extended to non-smooth energies such as the total variation on graphs that was defined over the continuous domain by Gilboa et al. [39] and over the discrete domain by Zhou and Scholkopf [78], [79]. Elmoataz et al. [34] considered a larger class of non-smooth energies involving a $p$-laplacian for $p<2$. Peyré replaced these non-linear flows on graphs by a non-iterative thresholding in a non-local spectral basis [62].

These graph-based regularizations were used to solve general inverse problems such as image deblurring [49], [21], inpainting of thin holes and removal of texture irregularities [41]. Moreover, Peyré et al [63] extended the total variation on non-local graphs to solve arbitrary inverse problems such as inpainting, super-resolution and compressive sampling. The graph-based regularizations are adaptive since the graph depends on the image, in other words, the graph is directly estimated from the measurements. Recently, for grey-scale image deblurring with Gaussian noise, Lou et al [50] used a preprocessed image obtained by applying the Wiener filter, instead of $\mathrm{f}$, to construct the weights.

Let us review the nonlocal differential operators over graphs and convex nonlocal functionals proposed by Gilboa and Osher [41]. Let $u: \Omega \rightarrow \mathbb{R}$ be a function, and $w: \Omega \times \Omega \rightarrow \mathbb{R}$ be a nonnegative and symmetric weight function. The nonlocal gradient vector $\nabla_{w} u: \Omega \times \Omega \rightarrow \mathbb{R}$ is $\left(\nabla_{w} u\right)(x, y):=(u(y)-u(x)) \sqrt{w(x, y)}$. Hence, the norm of the nonlocal gradient of $u$ at $x \in \Omega$ is defined as

$$
\left|\nabla_{w} u\right|(x):=\sqrt{\int_{\Omega}(u(y)-u(x))^{2} w(x, y) d y} .
$$

The nonlocal divergence $\operatorname{div}_{w} \vec{v}: \Omega \rightarrow \mathbb{R}$ of the vector $\vec{v}: \Omega \times \Omega \rightarrow \mathbb{R}$ is defined as the adjoint of the nonlocal gradient

$$
\left(\operatorname{div}_{w} \vec{v}\right)(x):=\int_{\Omega}(v(x, y)-v(y, x)) \sqrt{w(x, y)} d y .
$$

Based on these nonlocal operators, they introduced nonlocal regularizing functionals of the general form

$$
\Psi(u)=\int_{\Omega} \phi\left(\left|\nabla_{w} u\right|^{2}\right) d x
$$

where $s \mapsto \phi(s)$ is a positive function, convex in $\sqrt{s}$, and $\phi(0)=0$. By taking $\phi(s)=\sqrt{s}$, they proposed the nonlocal TV regularizer (NL/TV)

$$
\Psi^{N L / T V}(u)=\int_{\Omega}\left|\nabla_{w} u\right| d x=\int_{\Omega} \sqrt{\int_{\Omega}(u(y)-u(x))^{2} w(x, y) d y} d x,
$$

which corresponds in the local two dimensional case to $\Psi^{T V}(u)=\int_{\Omega}|\nabla u| d x$.

Inspired by these works, we propose in the next section nonlocal versions of Ambrosio-Tortorelli and Shah approximations to the MS regularizer for color image restoration such as deblurring-denoising in the presence of Gaussian noise or impulse noise, inpainting with large missing parts, superresolution of a single image, and image demosaicing, by extending the 1-dimensional nonlocal operators to 3-dimensional ones. This is also continuation or nonlocal extension of the work by Bar et al. [7], [9], first to propose the use of local Mumford-Shah-like approximations to color image deblurring-denoising in the presence of noise.

\section{Proposed NONLOCAL MUMFORD-ShaH REgUlarizers}

We propose the following nonlocal Mumford-Shah regularizers (NL/MS) by applying the nonlocal operators to the multichannel approximations of the MS regularizer

$$
\Psi^{N L / M S}(u, v)=\beta \int_{\Omega} v^{2} \phi\left(\left\|\nabla_{w} u\right\|^{2}\right) d x+\alpha \int_{\Omega}\left(\epsilon|\nabla v|^{2}+\frac{(v-1)^{2}}{4 \epsilon}\right) d x
$$


where $u: \Omega \rightarrow \mathbb{R}^{3}, v: \Omega \rightarrow[0,1], \phi(s)=s$ or $\phi(s)=\sqrt{s}$ correspond to the nonlocal versions of $\mathrm{MSH}^{1}$ and MSTV regularizers, so called NL/MSH ${ }^{1}$ and NL/MSTV, respectively:

$$
\begin{aligned}
& \Psi^{N L / M S H^{1}}(u, v)=\beta \int_{\Omega} v^{2}\left\|\nabla_{w} u\right\|^{2} d x+\alpha \int_{\Omega}\left(\epsilon|\nabla v|^{2}+\frac{(v-1)^{2}}{4 \epsilon}\right) d x, \\
& \Psi^{N L / M S T V}(u, v)=\beta \int_{\Omega} v^{2}\left\|\nabla_{w} u\right\| d x+\alpha \int_{\Omega}\left(\epsilon|\nabla v|^{2}+\frac{(v-1)^{2}}{4 \epsilon}\right) d x,
\end{aligned}
$$

where $\left\|\nabla_{w} u\right\|: \Omega \rightarrow \mathbb{R}$ is defined as

$$
\left\|\nabla_{w} u\right\|(x):=\sqrt{\sum_{i=r, g, b}\left|\nabla u_{w}^{i}\right|^{2}(x)}:=\sqrt{\sum_{i=r, g, b} \int_{\Omega}\left(u^{i}(y)-u^{i}(x)\right)^{2} w(x, y) d y} .
$$

We apply these nonlocal MS regularizers to color image denoising, color image deblurring in the presence of Gaussian or impulse noise, color image inpainting, color image super-resolution, and moreover, to color filter array demosaicing by incorporating proper fidelity terms. Furthermore, for deblurring in the presence of impulse noise, we propose a preprocessing step to evaluate the weights $w$ based on the preprocessed image. In practice, we use the standard weight function $w$ at $(x, y) \in \Omega \times \Omega$ depending on an image $q: \Omega \rightarrow \mathbb{R}$;

$$
w(x, y)=\exp \left(-\frac{d_{a}(q(x), q(y))}{h^{2}}\right),
$$

which gives the similarity of the color values as well as of image features between two pixels $x$ and $y$ in the image $q$ that will be defined in each section. For a fixed pixel $x \in \Omega$, we use a search window $S(x)=\{y \in \Omega:|x-y| \leq r\}$, instead of $\Omega$, to compute $w(x, y)$.

Note that the nonlocal and nonconvex continuous models proposed in the following sections have not been analyzed theoretically; however, these formulations become well-defined in the discrete, finite differences case, but we prefer to present them in the continuous setting for simplicity.

\section{IMAGE RESTORATION WITH NL/MS REGULARIZERS}

\section{A. Color image deblurring and denoising}

Image blur and noise are the most common problems in photography, which can be especially significant in light limited situations, resulting in a ruined photograph. Image deblurring (or deconvolution) is the process of recovering a sharp image from an input image corrupted by blurring and noise, where the blurring is due to convolution with a known or unknown kernel; see [27], [44], [8], [9], [17]. Recently, new image denoising models [20], [21], [52], [71], [40], [41], [62], based on nonlocal image information, have been developed to better deal with textures. The degradation model for color image deblurring-denoising (or denoising) is

$$
f=k * u+n \quad\left(f_{i}=k * u_{i}+n_{i}, i=r, g, b\right)
$$

where $k$ is a (known) space-invariant blurring kernel, and $n$ is an additive Gaussian noise or impulse noise (saltand-pepper noise or random-valued impulse noise).

First, in the case of Gaussian noise model, the $L^{2}$-fidelity term led by the maximum likelihood estimation is commonly used:

$$
\Phi(f-k * u)=\int_{\Omega} \sum_{i}\left|f^{i}-k * u^{i}\right|^{2} d x .
$$

However, the quadratic data fidelity term considers the impulse noise, which might be caused by bit errors in transmissions or wrong pixels, as an outlier. So, for the impulse noise model, the $L^{1}$-fidelity term is more appropriate, due to its robustness of removing outlier effects [5], [59]; moreover, we consider the case of independent channels noise [9]:

$$
\Phi(f-k * u)=\int_{\Omega} \sum_{i}\left|f^{i}-k * u^{i}\right| d x .
$$


Thus, we design two types of total energies for color image deblurring-denoising, depending on the type of noise as follows:

$$
\begin{aligned}
& \text { Gaussian noise: } \quad E^{G}(u, v)=\frac{1}{2} \int_{\Omega} \sum_{i}\left|f^{i}-k * u^{i}\right|^{2} d x+\Psi^{N L / M S}(u, v) \\
& \text { Impulse noise: } \quad E^{I m}(u, v)=\int_{\Omega} \sum_{i}\left|f^{i}-k * u^{i}\right| d x+\Psi^{N L / M S}(u, v) .
\end{aligned}
$$

Preprocessing for the impulse noise model: To extend the nonlocal methods to the impulse noise case, we need a preprocessing step for the weight function $w$ since we cannot directly use the data $f$ to compute $w$. In other words, in the presence of impulse noise, the noisy pixels tend to have larger weights than the other neighboring points, so it's likely to keep the noise value at such pixel. Thus, we propose a simple algorithm to obtain a preprocessed image $\bar{g}$, which removes the impulse noise (outliers) as well as preserves the textures as much as we can. Basically, we use the median filter, well-known for removing impulse noise. However, for the deblurring-denoising model, if we apply one-step of the median filter, then the output may be too smoothed out. In order to preserve fine structures as well as to remove noise properly, we define a preprocessing method for the deblurring-denoising model inspired by the idea of Bregman iteration [15]. Thus, we propose the following algorithm to obtain a preprocessed image $\bar{g}$ that will be used only in the computation of the weight function $w$ :

$$
\begin{aligned}
& \text { Initialize }: r_{0}^{i}=0, g_{0}^{i}=0, i=r, g, b . \\
& \text { do (iterate } n=0,1,2, \ldots) \\
& \qquad \begin{aligned}
g_{n+1} & =\operatorname{median}\left(f+r_{n},\left[\begin{array}{ll}
a & a
\end{array}\right]\right) \\
r_{n+1} & =r_{n}+f-k * g_{n+1}
\end{aligned}
\end{aligned}
$$

while $\sum_{i}\left\|f^{i}-k * g_{n}^{i}\right\|_{1}>\sum_{i}\left\|f^{i}-k * g_{n+1}^{i}\right\|_{1}$

where $f$ is the given noisy-blurry data, and median $\left(f,\left[\begin{array}{ll}a & a\end{array}\right]\right)$ is the median filter of size $a \times a$ with input $f$. The preprocessed image $\bar{g}$ is a deblurred and denoised version of $f$, but it still includes some irregularities (or remaining impulse noise) that will be handled by constructing binary weights proposed for detecting and removing irregularities from texture in [41]. The image $\bar{g}$ will be used only in the computation of the weights $w$, while keeping $f$ in the data fidelity term, thus artifacts are not introduced by the median filter.

Characterization of minimizers: In this section we characterize the minimizers of the functionals formulated with the nonlocal regularizers, using [56], [72]. Assuming that a functional $\|\cdot\|$ on a subspace of $\left(L^{2}(\Omega)\right)^{3}$ is a seminorm, we can define the dual norm (where $\langle\cdot, \cdot\rangle$ denotes the $\left(L^{2}(\Omega)\right)^{3}$ inner product) of $f \in\left(L^{2}(\Omega)\right)^{3} \subset\left(L^{1}(\Omega)\right)^{3}$ as $\|f\|_{*}:=\sup _{\|\varphi\| \neq 0} \frac{\langle f, \varphi\rangle}{\|\varphi\|} \leq+\infty$, so that the usual duality $\langle f, \varphi\rangle \leq\|\varphi\|\|f\|_{*}$ holds for $\|\varphi\| \neq 0$. We define the functionals (here $K u:=k * u$ ),

$$
\begin{aligned}
F(u) & =\lambda \int_{\Omega} \sum_{i}\left|f^{i}-K u^{i}\right|^{2} d x+|u|_{N L / T V}, \\
G(u, v) & =\int_{\Omega} \sqrt{\sum_{i}\left|f^{i}-K u^{i}\right|^{2}+\eta^{2}} d x+\beta|u|_{N L / M S}+\alpha \int_{\Omega}\left(\epsilon|\nabla v|^{2}+\frac{(v-1)^{2}}{4 \epsilon}\right) d x, \\
G^{\prime}(u, v) & =\sum_{i} \int_{\Omega} \sqrt{\left|f^{i}-K u^{i}\right|^{2}+\eta^{2}} d x+\beta|u|_{N L / M S}+\alpha \int_{\Omega}\left(\epsilon|\nabla v|^{2}+\frac{(v-1)^{2}}{4 \epsilon}\right) d x,
\end{aligned}
$$

where $\lambda>0$, and $|u|_{N L / M S} \in\left\{|u|_{N L / M S H^{1}, v},|u|_{N L / M S T V, v}\right\}$ with

$$
\begin{aligned}
& |u|_{N L / T V}=\int_{\Omega}\left\|\nabla_{w} u\right\|(x) d x, \quad|u|_{N L / M S H^{1}, v}=\sqrt{\int_{\Omega} v^{2}(x)\left\|\nabla_{w} u\right\|^{2}(x) d x}, \\
& |u|_{N L / M S T V, v}=\int_{\Omega} v^{2}(x)\left\|\nabla_{w} u\right\|(x) d x .
\end{aligned}
$$

Note that the regularizers $|u|_{N L / T V},|u|_{N L / M S H^{1}, v}$ and $|u|_{N L / M S T V, v}$ are semi-norms (Appendix II). In addition, 
we modified the regularizing functional $|u|_{N L / M S H^{1}, v}$; the square-root term replaces the original term,

$$
\int_{\Omega} v^{2}(x)\left\|\nabla_{w} u\right\|^{2}(x) d x
$$

of our model. It is introduced here to enable the characterization of minimizers below, but the numerical calculations utilize the original formulation.

Proposition. 1. Let $K:\left(L^{2}(\Omega)\right)^{3} \rightarrow\left(L^{2}(\Omega)\right)^{3}$ be a linear bounded blurring operator with adjoint $K^{*}$ and let $F$ be the associated functional. Then

(1) $\left\|K^{*} f\right\|_{*} \leq \frac{1}{2 \lambda}$ if and only if $u \equiv 0$ is a minimizer of $F$,

(2) Assume that $\frac{1}{2 \lambda}<\left\|K^{*} f\right\|_{*}<\infty$. Then $u$ is a minimizer of $F$ if and only if $\left\|K^{*}(f-K u)\right\|_{*}=\frac{1}{2 \lambda}$ and $\left\langle u, K^{*}(f-K u)\right\rangle=\frac{1}{2 \lambda}|u|_{N L / T V}$,

where $\|\cdot\|_{*}$ is the corresponding dual norm of $|\cdot|_{N L / T V}$. We omit the proof of Proposition. 1. since it is similar with the proofs of Corollaries 2.5 and 2.7 from [72].

Proposition. 2. Let $K:\left(L^{2}(\Omega)\right)^{3} \rightarrow\left(L^{2}(\Omega)\right)^{3}$ be a linear bounded blurring operator with adjoint $K^{*}$ and let $G$ be the associated functional. If $(u, v)$ is a minimizer of $G$ with $v \in[0,1]$, then

$$
\left\|K^{*} \frac{f-K u}{\sqrt{\sum_{i}\left(f^{i}-K u^{i}\right)^{2}+\eta^{2}}}\right\|_{*}=\beta \text { and }\left\langle K^{*} \frac{f-K u}{\sqrt{\sum_{i}\left(f^{i}-K u^{i}\right)^{2}+\eta^{2}}}, u\right\rangle=\beta|u|_{N L / M S},
$$

where $\|\cdot\|_{*}$ is the corresponding dual norm of $|\cdot|_{N L / M S}$. The proof of Proposition. 2. is given in Appendix II. Note that by replacing $G$ by $G^{\prime}$ in Proposition. 2, we have the conclusion such as

$$
\sum_{i}\left\langle K^{*} \frac{f^{i}-K u^{i}}{\sqrt{\left(f^{i}-K u^{i}\right)^{2}+\eta^{2}}}, u^{i}\right\rangle=\beta|u|_{N L / M S}
$$

where $\langle\cdot, \cdot\rangle$ is $L^{2}(\Omega)$ inner product.

\section{B. Color image inpainting}

Image inpainting, also known as image interpolation, is the process of reconstructing lost or corrupted parts of an image, that is an interesting and important inverse problem with many applications such as removal of scratches in old photos, removal of overlaid text or graphics, filling-in missing blocks in unreliably transmitted images. Nontexture image inpainting has received considerable interest since the pioneering paper by Masnou and Morel [54], [55] who proposed variational principles for image disocclusion. A recent wave of interest in inpainting has also started from [11], where applications in the movie industry, video and art restoration were unified. These authors proposed nonlinear partial differential equations for non-texture inpainting. Moreover, many contributed works have been proposed for the solution of this interpolation task based on (a) diffusion and transport PDE/variational principle [23], [13], [24], [26], [25], [35], [68], [63], [4], (b) exemplar region fill-in [32], [75], [12], [31], [61], [29], (c) compressive sensing [33], [70]. Inpainting corresponds to the operation $H$ of losing pixels from an image, i.e. the observed data $f$ is given by

$$
f=u \quad \text { on } \quad \Omega-D
$$

where $D=D^{0}$ is the region where the input data $u$ has been damaged. Thus, inspired from [26], we propose the total energy functional for color image inpainting as

$$
E^{I n p}(u, v)=\frac{1}{2} \int_{\Omega} \lambda_{D}(x) \sum_{i}\left|f^{i}-u^{i}\right|^{2} d x+\Psi^{N L / M S}(u, v),
$$

where $\lambda_{D}(x)=0$ if $x \in D$ and $\lambda_{D}(x)>0$ if $x \in \Omega-D$. In addition, we update the weights $w$ only in the damaged region $D^{0}$ at every $m$ th iteration for $u$ using the patch distance:

$$
d_{a}^{R}(u(x), u(y))=\int_{\Omega-R} G_{a}(t)\|u(x+t)-u(y+t)\|^{2} d t,
$$


where $R \subset D^{0}$ is an un-recovered region (still missing region). Therefore, the missing region $D^{0}$ is recovered by the following iterative algorithm, producing the un-recovered regions $D^{i}, i=0,1,2, \ldots$, with $D^{0} \supset D^{1} \supset D^{2} \supset \cdots$ :

1. Compute weights $w$ for $x \in \Omega$ s.t. $P(x) \bigcap\left(\Omega-D^{0}\right) \neq 0$ with $d_{a}^{D^{0}}\left(u^{0}(x), u^{0}(y)\right), u^{0}=f$, and a patch $P(x)$ centered at $x$.

2. Iterate $n=1,2, \ldots$ to get a minimizer $(u, v)$ starting with $u=u^{0}$ :

a. For fixed $u$, update $v$ in $\Omega$ to get $v^{n}$.

b. For fixed $v$, update $u$ in $\Omega$ to get $u^{n}$ with a recovered region $\Omega-D^{n} \supset \Omega-D^{0}$ : at every $m$ th iteration, update weights $w$ only in $x \in D^{0}$ s.t. $P(x) \bigcap\left(\Omega-D^{n, m}\right) \neq 0$ with

$$
d_{a}^{D^{n, m}}(u(x), u(y))
$$

where $D^{n, m}$ is an un-recovered region in $D^{0}$ until $m$ th iteration with $D^{n, m} \supset D^{n, 2 m} \supset \cdots \supset D^{n, n}=D^{n}$.

\section{Color image super-resolution}

Super-resolution corresponds to the recovery of a high resolution image from a filtered and down-sampled image. It is usually applied to a sequence of images in video; see [53], [37], [36], [30], [64]. We consider here a simpler problem of increasing the resolution of a single still image, and the observed data $f$ is given by

$$
f^{i}=D_{k}\left(h * u^{i}\right), \quad i \in r, g, b
$$

where $h$ is a low-pass filter, $D_{k}: \mathbb{R}^{n} \rightarrow \mathbb{R}^{p}$ (with $p=\left[\frac{n}{k^{2}}\right]$ where $[q]$ is the integer part of $q$ ) is the down-sampling operator by a factor $k$ along each axis. We want to recover a high resolution image $u \in\left(\mathbb{R}^{n}\right)^{3}$ by minimizing

$$
E^{S u p}(u, v)=\frac{1}{2} \int_{\Omega} \sum_{i}\left|f^{i}-D_{k}\left(h * u^{i}\right)\right|^{2} d x+\Psi^{N L / M S}(u, v) .
$$

In addition, we use a super-resolved image $\bar{g} \in\left(\mathbb{R}^{n}\right)^{3}$ obtained by bicubic interpolation of $f \in\left(\mathbb{R}^{p}\right)^{3}$ only for the computation of the weights $w$.

\section{Color filter array demosaicing}

A demosaicing algorithm is to reconstruct a full color image from the incomplete color samples output from an image sensor overlaid with a color filter array (CFA). A color filter array is a mosaic of color filters in front of the image sensor, and we here use the Bayer filter [10] that has alternating green $(G)$ and red (R) filters for odd rows and alternating blue (B) and green $(\mathrm{G})$ filters for even rows. Since each pixel of the sensor is behind a color filter, the output is an array of pixel values, each indicating a raw intensity of one of the three filter colors. Thus, an algorithm is needed to estimate for each pixel the color levels for all color components, rather than a single component; see [3], [48], [42], [43], [51], [45], [22]. In this variational framework, we consider the observed data $f$ such as

$$
f^{i}=B^{i} \cdot u^{i}, \quad i \in\{r, g, b\}
$$

where $\cdot$ is the pointwise product, and $B^{i}: \mathbb{R}^{n} \rightarrow \mathbb{R}^{n}$ is the down-sampling operator; $B^{g}$ has alternating 1 and 0 values for odd rows and alternating 0 and 1 values for even rows, $B^{r}$ has alternating 0 and 1 values for odd rows and only 0 values for even rows, $B^{b}$ has only 0 values for odd rows and alternating 1 and 0 values for even rows. We propose the following minimization problem to recover a full color image $u$ :

$$
E^{D e m o}(u, v)=\frac{1}{2} \int_{\Omega} \sum_{i}\left|f^{i}-B^{i} \cdot u^{i}\right|^{2} d x+\Psi^{N L / M S}(u, v) .
$$

Moreover, we use the interpolated image obtained by applying Hamilton-Adams algorithm [3] to the green channel and bilinear interpolation to $r-g$ and $b-g$, in order to compute the initial weight function $w$. In the HamiltonAdams method, the evaluation of the gradient at the missing green pixel is corrected by the second order derivatives of the red or blue channels. In addition, as in [22], in order to gradually correct the erroneous structures and artifacts of initial color image $u_{0}$, we also proceed by an iterative strategy refining at each step the similarity search by reducing the value of parameter $h$ in the weights as: 
- Initialize $u=u_{0}$ with an interpolated image with Hamilton-Adams algorithm.

- Iterate for $h$ (e.g. $h=\{16,8,4\})$ :

a. Construct the weight function $w=w_{u}$ using the image $u$.

b. Compute a minimizer $(u, v)$ by minimizing the functional $E^{D e m o}(u, v)$.

We refer to [22] using NL-means for prior relevant work.

\section{NUMERICAL DisCRETIZATIONS}

Minimization of the proposed functionals (2)-(6): $E^{G}, E^{I m}, E^{I n p}, E^{S u p}, E^{D e m o}$ in $u$ and $v$ is carried out using the Euler-Lagrange equations (computed in Appendix III)

$$
\begin{aligned}
\frac{\partial E^{G, I m, I n p, \text { Sup }, \text { Demo }}}{\partial v} & =2 \beta v \phi\left(\left\|\nabla_{w} u\right\|^{2}\right)-2 \epsilon \alpha \Delta v+\alpha\left(\frac{v-1}{2 \epsilon}\right)=0, \\
\frac{\partial E^{G}}{\partial u} & =\tilde{k} *(k * u-f)+L^{N L / M S} u=0, \\
\frac{\partial E^{I m}}{\partial u} & =\tilde{k} * \operatorname{sign}(k * u-f)+L^{N L / M S} u=0, \\
\frac{\partial E^{I n p}}{\partial u} & =\lambda_{D}(u-f)+L^{N L / M S} u=0, \\
\frac{\partial E^{S u p}}{\partial u} & =\tilde{h} *\left(D_{k}^{T}\left(D_{k}(h * u)-f\right)\right)+L^{N L / M S} u=0, \\
\frac{\partial E^{\text {Demo }}}{\partial u} & =B \cdot(B \cdot u-f)+L^{N L / M S} u=0,
\end{aligned}
$$

where $\tilde{k}(x)=k(-x), \tilde{h}(x)=h(-x), D_{k}^{T}:\left(\mathbb{R}^{p}\right)^{3} \rightarrow\left(\mathbb{R}^{n}\right)^{3}$ is the transpose of $D_{k}$ i.e. the up-sampling operator, and (see Appendix II)

$$
\begin{aligned}
L^{N L / M S} u= & -2 \int_{\Omega}\{(u(y)-u(x)) w(x, y) \\
\cdot & {\left[\left(v^{2}(y) \phi^{\prime}\left(\left\|\nabla_{w} u\right\|^{2}(y)\right)+v^{2}(x) \phi^{\prime}\left(\left\|\nabla_{w} u\right\|^{2}(x)\right)\right]\right\} d y . }
\end{aligned}
$$

To solve two Euler-Lagrange equations simultaneously, the alternate minimization approach is applied. Note that since the energy functionals are not convex in the joint variable $(u, v)$, we may compute only a local minimizer. However, this is not a drawback in practice, since the initial guess for $u$ in our algorithm is the data $f$ (except for the super-resolution problem). Due to its simplicity, we use Gauss-Seidel scheme for $v$, and an explicit scheme for $u$ using gradient descent method, leading to the following iterative algorithm:

- Initialization: $u^{0}=f, v^{0}=1$.

- Iterate $n=0,1,2, \ldots$, until $\left(\left\|u^{n+1}-u^{n}\right\|_{2}<\eta\left\|u^{n}\right\|_{2}\right)$.

1. Solve the equation for $v^{n+1}$ using Gauss-Seidel scheme:

$$
\left(2 \beta \phi\left(\left\|\nabla_{w} u^{n}\right\|^{2}\right)+\frac{\alpha}{2 \epsilon}-2 \epsilon \alpha \triangle\right) v^{n+1}=\frac{\alpha}{2 \epsilon}
$$

2. Set $u^{n+1,0}=u^{n}$ and solve for $u^{n+1}$ by iterating on $l$ :

$$
u^{n+1, l+1}=u^{n+1, l}+d t \cdot G\left(u^{n+1, l}, v^{n+1}\right)
$$

where $G=-\partial E / \partial u$.

Here $\eta$ is a small positive constant. The basic discretizations are explained next. Let $u_{k}^{\sigma}$ denote the value of a pixel $k$ in the image $(1 \leq k \leq N)$ with channel $\sigma$ (i.e. the discretized version of $u^{\sigma}(x)$ defined on $\Omega$ ), and let $p_{k, l}^{\sigma}$ be the discretized version of $p^{\sigma}(x, y)$ with $x, y \in \Omega$. And $w_{k, l}$ is the sparsely discrete version of $w=w(x, y): \Omega \times \Omega \rightarrow \mathbb{R}$. We use the neighbors set $l \in N_{k}$ defined as $l \in N_{k}:=\left\{j: w_{k, l}>0\right\}$. Then we have $\nabla_{w d}$ and $d i v_{w d}$, the discretizations of $\nabla_{w}$ and $d i v_{w}$, respectively given by [41]

$$
\begin{aligned}
\nabla_{w d}\left(u_{k}^{\sigma}\right) & :=\left(u_{l}^{\sigma}-u_{k}^{\sigma}\right) \sqrt{w_{k, l}}, \quad l \in N_{k}, \\
\operatorname{div}_{w d}\left(p_{k, l}^{\sigma}\right) & :=\sum_{l \in N_{k}}\left(p_{k, l}^{\sigma}-p_{l, k}^{\sigma}\right) \sqrt{w_{k, l}} .
\end{aligned}
$$



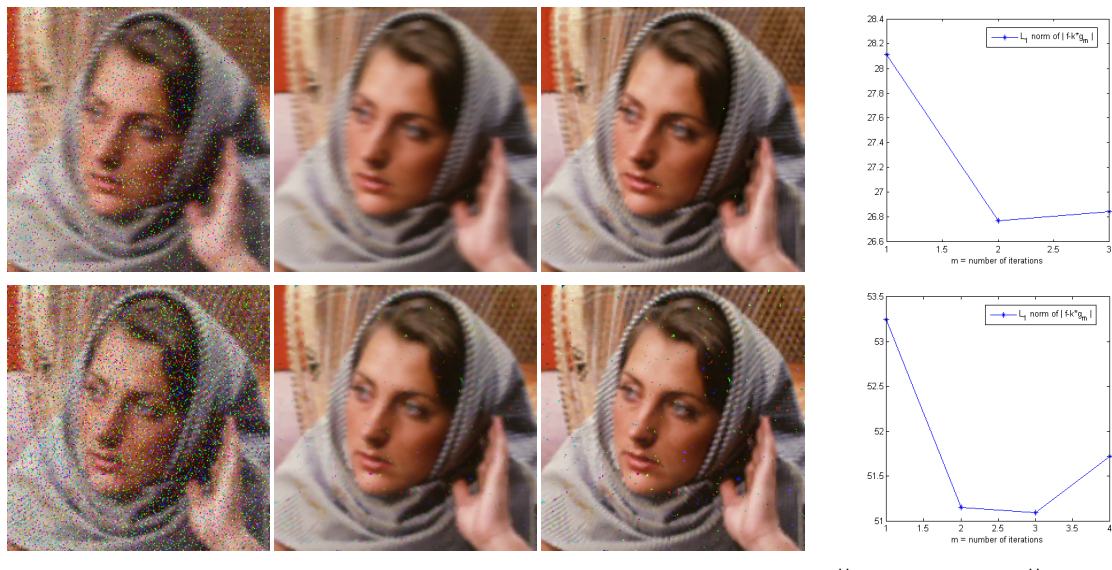

$$
\text { noisy-blurry } f
$$

$g_{1}$

$\bar{g}$

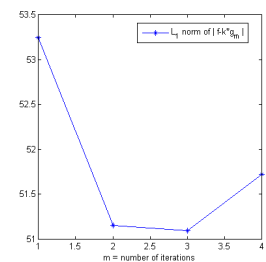

$\left\|f-k * g_{m}\right\|_{1}$ vs $m$

Fig. 1. Preprocessed images $\bar{g}$ in the presence of Random-valued impulse noise. Data $f$ : (Top) motion blur kernel with length of 8 and orientation 0 , noise density $d=0.1$, (Bottom) motion blur kernel with length of 4 and orientation 0 , noise density $d=0.2 . \bar{g}=g_{2}$ (top), $\bar{g}=g_{3}$ (bottom) with $3 \times 3$ median filter.

Moreover, the magnitude of $p_{k, l}^{\sigma}$ at $k$ is $\left|p^{\sigma}\right|_{k}=\sqrt{\sum_{l}\left(p_{k, l}^{\sigma}\right)^{2}}$, thus the discretization of $\left\|\nabla u_{w}\right\|^{2}(x)$ was done as

$$
\left\|\nabla u_{w d}\right\|_{k}^{2}=\sum_{\sigma \in\{r, g, b\}}\left|\nabla_{w d} u^{\sigma}\right|_{k}^{2}=\sum_{\sigma \in\{r, g, b\}} \sum_{l}\left(u_{l}^{\sigma}-u_{k}^{\sigma}\right)^{2} w_{k, l} .
$$

Basically, we construct the weight function $w_{k, l}$, following the algorithm in [40]: for each pixel $k$, (1) take a patch $P_{k}$ around a pixel $k$, compute the distances $\left(d_{a}\right)_{k, l}$ (a discretization of $d_{a}$ ) to all the patches $P_{l}$ in the search window $l \in S(k)$, and construct the neighbors set $N_{k}$ by taking the $m$ most similar and the four nearest neighbors of the pixel $k,(2)$ compute the weights $w_{k, l}, l \in N_{k}$ and set to zero for all the other connections $\left(w_{k, l}=0, l \notin N_{k}\right)$, (3) set $w_{k, l}=w_{l, k}, l \in N_{k}$. For deblurring in the presence of impulse noise, we used $m=5$, so a maximum of up to $2 m+4$ neighbors for each pixel is allowed, and $5 \times 5$ pixel patches with $a=10$, a search window of size $11 \times 11$. The complexity of computing the weights using this algorithm is $N \times$ Window $_{\text {size }} \times\left(\right.$ Patch $_{\text {size }} \times$ Channal $\left._{\text {size }}+\log m\right)$. Thus, in this case, we need $121 \times(25 \times 3+2.5) \approx 9619$ operations per pixel. Note that, when we use a preprocessed image $\bar{g}$ to compute $w$ in the impulse noise model, we construct the weight function $w$ with the binary values of 0 or $1[41]$.

\section{EXPERIMENTAL RESULTS AND COMPARISONS}

The nonlocal MS regularizers proposed here, NL/MSH ${ }^{1}$ and NL/MSTV, are tested on several color images corrupted by different blur kernels or different noise types, on color images with missing parts, on sub-sampled color images, as well as on incomplete color samples outputs. We mostly compare them with their local versions [9]. For deblurring in the presence of impulse noise, we also present results with TV [17] and NL/TV [50] models because our work is the first trial to extend nonlocal methods to impulse noise model. For denoising or deblurring in the presence of Gaussian noise and super-resolution, we only present the results of MSTV and NL/MSTV because $\mathrm{NL} / \mathrm{MSH}^{1}$ produces smoother images leading to similar PSNR values with MSTV.

First, in Figures 1-3, we recover blurred images contaminated by random-valued impulse noise with noise density $d=0.1$ or $d=0.2$. Fig. 1 present the preprocessed images $\bar{g}$ obtained by iterative median filter of size $3 \times 3$, and the corresponding energies $\left\|f-k * g_{m}\right\|_{1}$ vs $m$. As $m$ increases, the image $g_{m}$ gets deblurred and denoised to some extent where the energy has a minimum. Thus, the image having the minimum energy value is chosen as a preprocessed image $\bar{g}$, but it still contains some impulse noise or artifacts. However, the recovered images using nonlocal regularizers in Fig. 2 and 3 show that the artifacts on the preprocessed images are well handled when constructing weights, thus they do not influence the final recovered images. Hence, by computing the weight function $w$ based on the preprocessed images $\bar{g}$, all the nonlocal regularizers recover textures better and reduce the artifacts by blur kernel (especially on the face and hand), providing cleaner images as well as higher PSNR values. Fig. 4 provides the edge set $v$ of local or nonlocal MS regularizers, concurrently obtained during the restoration process in Figures 2-3. 


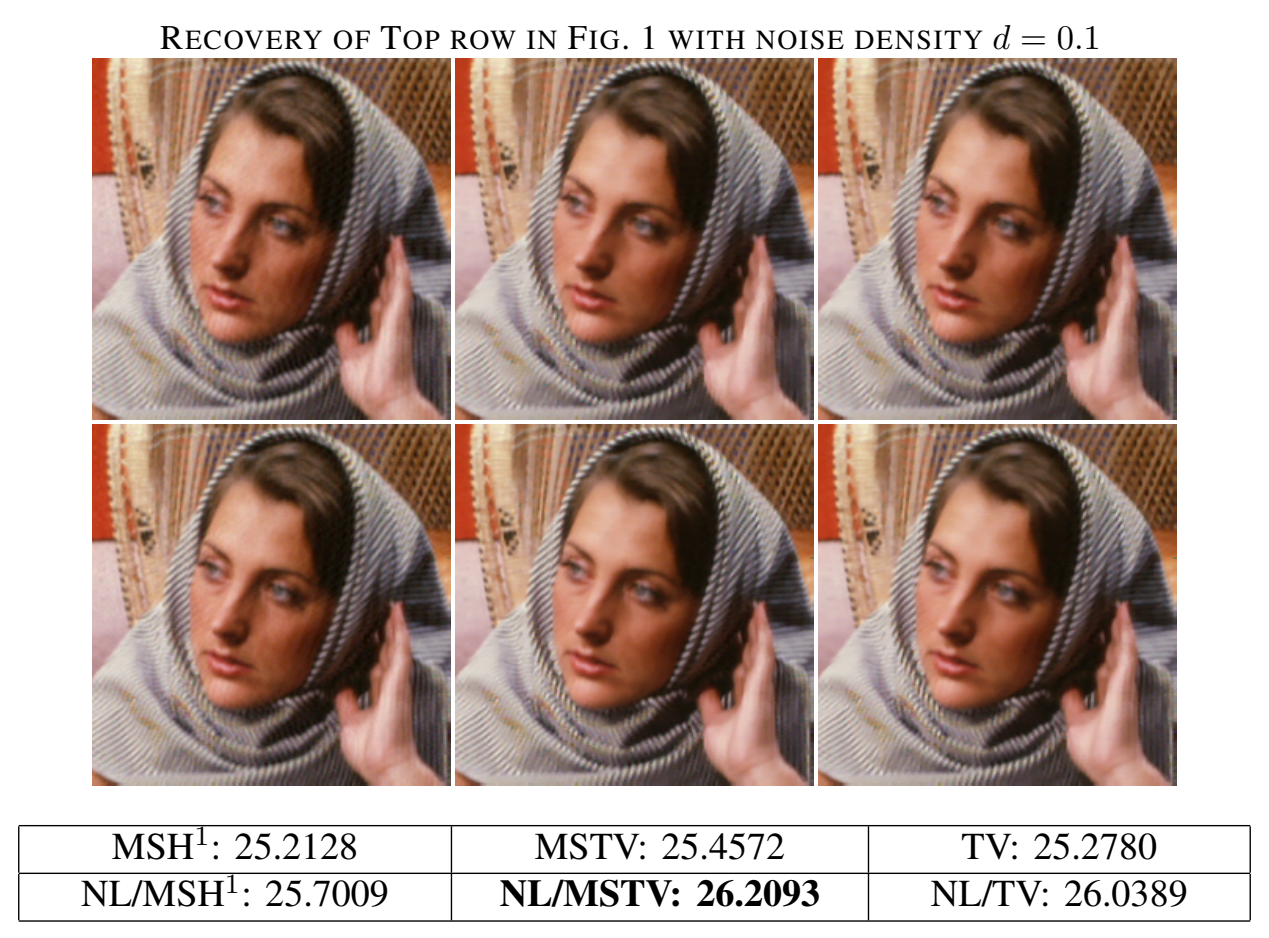

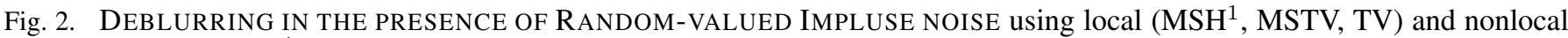
regularizers (NL/MSH ${ }^{1}$, NL/MSTV, NL/TV). PSNR: $f: 16.9528, \bar{g}: 21.7047$.

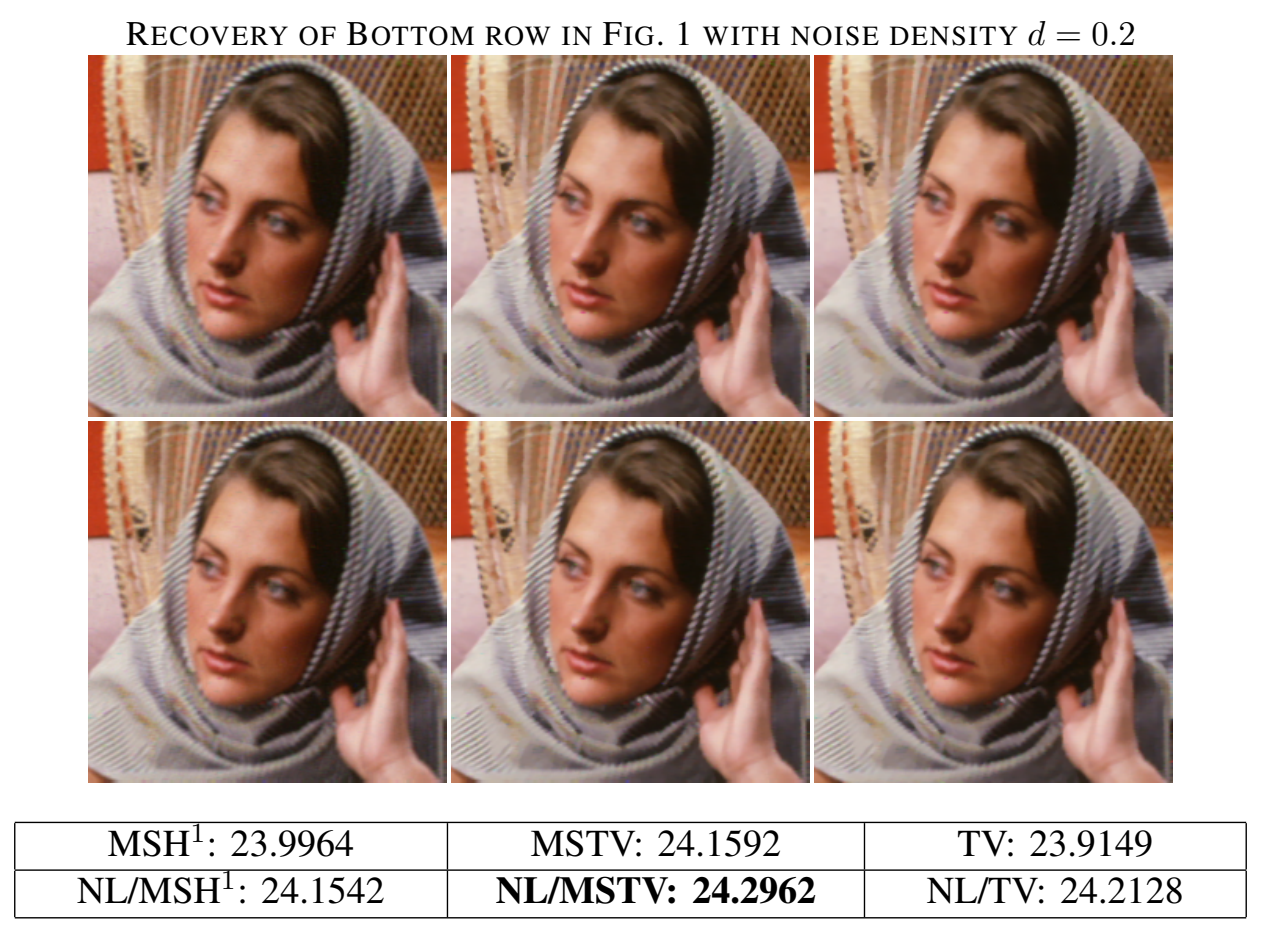

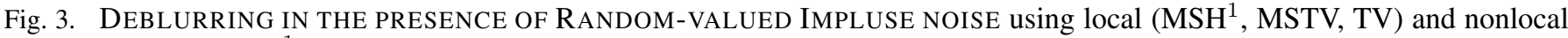
regularizers (NL/MSH ${ }^{1}$, NL/MSTV, NL/TV). PSNR: $f: 14.9649, \bar{g}: 22.3935$.

In Figures 5-7, we recover blurred images contaminated by salt-and-pepper noise with various high noise densities $d=0.3,0.4,0.5$. In Fig. 5 , we present the preprocessed images $\bar{g}$ obtained by iterative median filters with different sizes, and the images obtained by one-step median filters for comparison. First, we observe that the images using iterative median filters are deblurred and denoised versions of noisy-blurry data. For the case of $d=0.3$ and 0.4 $(d=0.5)$, we choose the preprocessed images with iterative median filter of size $7 \times 7(9 \times 9)$, since the ones 


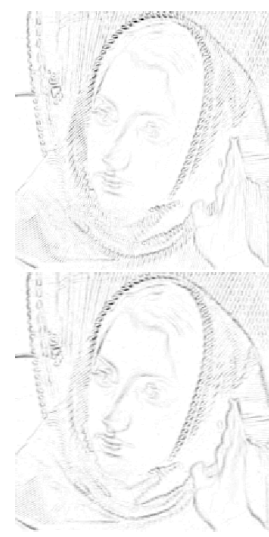

(a) $\mathrm{MSH}^{1}$

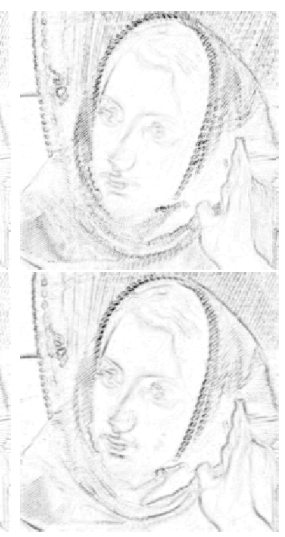

(b) $\mathrm{NL} / \mathrm{MSH}^{1}$

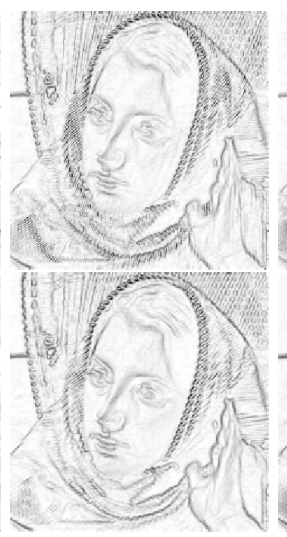

(c) MSTV

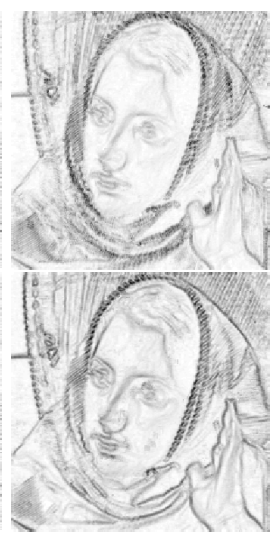

(d) NL/MSTV

Fig. 4. $\quad$ Edge set $v$ obtained during the restoration process using $\mathrm{MSH}^{1}, \mathrm{NL} / \mathrm{MSH}^{1}$, MSTV, NL/MSTV in Fig. 2 (Top row) and 3 (Bottom row).

with smaller median filters give more severe artifacts, that can influence the weights, tending to remain in the final recovered images. We show the recovered images using local and nonlocal regularizers in Fig. 7 and the corresponding PSNR values in Fig. 6. Visually and according to PSNR values, the nonlocal regularizers recover the degraded images better than the local ones. Specifically, NL/MSH ${ }^{1}$ reduces ringing artifacts appeared in the recovered images with $\mathrm{MSH}^{1}$; NL/MSTV and NL/TV reduce the staircase effects appeared in the results obtained by the corresponding local models, and suppress less the texture. Furthermore, we observe that the (local or nonlocal) MSTV regularizers are more robust than the (local or nonlocal) TV regularizers, resulting in clearer restoration, which might be caused by the fact that they do not suppress the edge part as much as TV regularizers do, and additionally yielding image edge sets $v$. Comparing with the (local or nonlocal) $\mathrm{MSH}^{1}$ regularizers, the (local or nonlocal) MSTV regularizers produce cleaner images, despite the cartoon-like restored images especially in the case of high noise density.

Moreover, in Fig. 8, we test on the Girl images corrupted by random-valued impulse noise; either high blur and noise with $d=0.3$ (2, 3rd rows) or low blur and higher noise with $d=0.4\left(3,4\right.$ th rows). In the first case, $\mathrm{NL} / \mathrm{MSH}^{1}$ reduces very much the ringing effect especially appeared on the cloth part with $\mathrm{MSH}^{1}$, providing cleaner image, and NL/TV gives much better restored image than TV, leading to much higher PSNR. Even though MSTV gives desired recovered image already, NL/MSTV additionally reduces the staircase effect on the image with MSTV, resulting in more realistic image as well as higher PSNR. Both NL/MSTV and NL/TV give very well recovered images visually and according to PSNR values. In the second case with less blur but more noise, NL/MSTV and $\mathrm{NL} / \mathrm{TV}$ give sharper images than corresponding local ones as well as the $\mathrm{MSH}^{1}$ regularizers.

In Fig. 10, we only test MSTV and NL/MSTV for the Gaussian noise model. For the noisy Lena image corrupted by Gaussian noise with noise density $d=0.02$, NL/MSTV recovers texture much better (hat part) and provides cleaner edges, while MSTV smoothes out many details and has noisy edges. In addition, for the noisy-blurry house and castle images, NL/MSTV gives cleaner and sharper restored images, leading to higher PSNR values.

In Figures 11-13 we use the NL/MS regularizers to recover images with texture and large missing portions. In Fig. 11, we present the process of inpainting, and final recovered images using NL/MS regularizers. We can easily see that both nonlocal regularizers recover the missing parts very well, and moreover NL/MSTV gives slightly better result than NL/MSH ${ }^{1}$ according to PSNR even though they visually seem to produce very similar results. However, in Fig. 12 with a real image, NL/MSH ${ }^{1}$ gives slightly higher PSNR values, especially recovering better the part damaged by the rectangle in the bottom. Both NL/MS regularizers recover the missing parts gradually, as seen in Fig. 13, while local MS regularizers fail to recover them.

In Figures 14-15, we recover an image filtered with a low-pass filter and then sub-sampled, using MSTV and NL/MSTV. As seen in both recovered images and edge sets $v$, NL/MSTV (incorporating weights based on the interpolated image $\bar{g}$ ) provides clearer edges, leading to much higher PSNR, while MSTV produces some artifacts especially on the edges. For comparison, we apply Lucy-Richardson deconvolution iterative algorithm to the blurry image $\bar{g}$, but it produces severe artifacts and provides lower PSNR than for $\bar{g}$ itself as well as for the recovered 


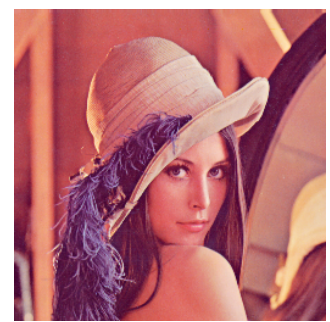

original

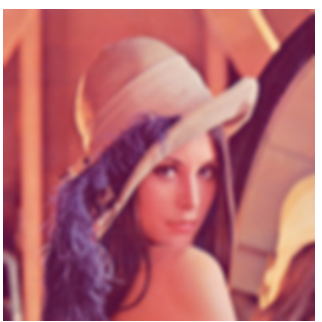

blurry image

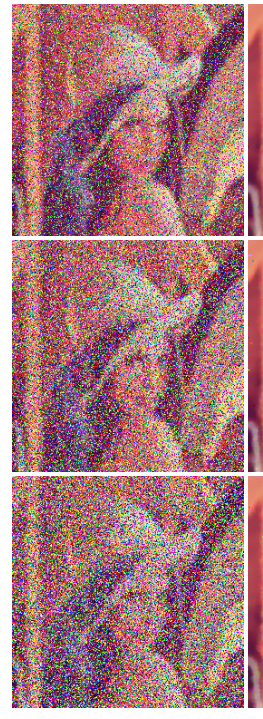

(a)

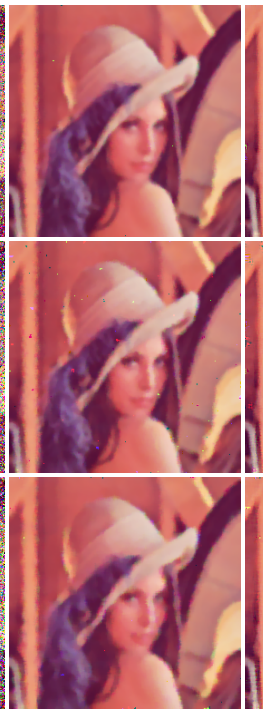

(b)

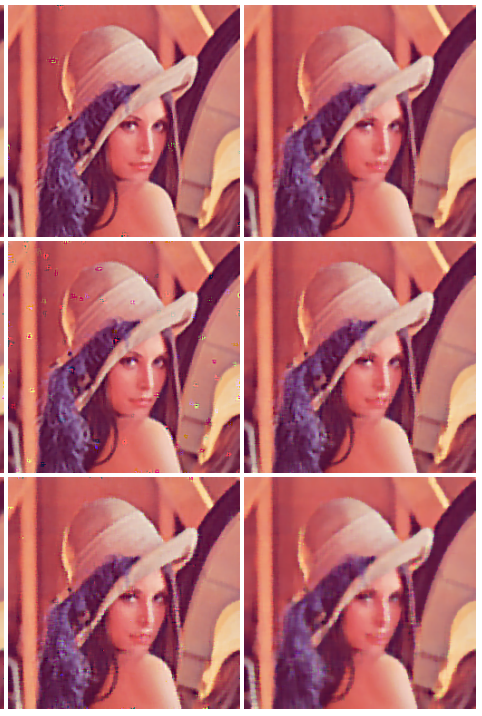

(c)

(d)
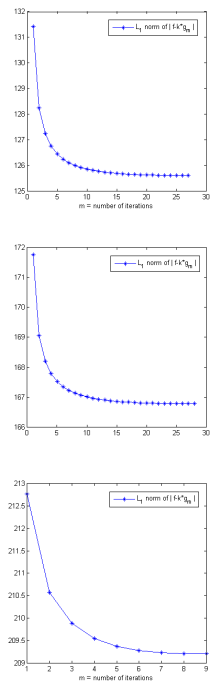

(e)

\begin{tabular}{|c|c|}
\hline (a) data & (c)-(e) iterative median filter of size $m \times m$ \\
\hline SP, $d=0.3$ & (c) $m=5$, (d)-(e) $m=7$ with corresponding energy \\
\hline SP, $d=0.4$ & (c) $m=5$, (d)-(e) $m=7$ with corresponding energy \\
\hline SP, $d=0.5$ & (c) $m=7$, (d)-(e) $m=9$ with corresponding energy \\
\hline
\end{tabular}

Fig. 5. Preprocessed images using iterative median filter. (a) noisy-blurry data blurred with Gaussian blur kernel with $\sigma_{b}=2$ and then contaminated by Salt-and-Pepper noise with noise density $d$. (b) recovered image using one-step median filter of size $5 \times 5$ (1, 2nd rows) or $7 \times 7$ (3rd row). (c)-(e) recovered image using iterative median filter and plot of energy $\left\|f-k * g_{m}\right\|_{1}$ vs $m$.

\begin{tabular}{|c||c|c|c|}
\hline noisy density & $d=0.3$ & $d=0.4$ & $d=0.5$ \\
\hline data $f$ & 10.3172 & 9.1047 & 8.1583 \\
TV & 28.4720 & 28.0494 & 27.2286 \\
MSH $^{1}$ & 28.4402 & 28.1353 & 27.1543 \\
MSTV & 28.8540 & 28.4862 & 27.5305 \\
NL/TV & 28.7825 & 28.5808 & 27.7406 \\
NL/MSH & 28.7334 & 28.2866 & 27.4064 \\
NL/MSTV & $\mathbf{2 9 . 4 6 4 1}$ & $\mathbf{2 8 . 7 9 7 7}$ & $\mathbf{2 7 . 9 6 4 0}$ \\
preprocessed $\bar{g}$ & 26.1987 & 25.7309 & 24.6567 \\
\hline
\end{tabular}

Fig. 6. PSNR values of recovered images from the data in Fig. 5. Preprocessed images $\bar{g}$ are given in Fig. 5 (d). Recovered images are given in Fig. 7.

images using MSTV and NL/MSTV.

In Figures 16-19, we reconstruct full color images from the incomplete color samples outputs by Bayer color filter, using NL/MS regularizers with the decreasing sequences of $h$. In all the examples, we use the initial color image $u_{0}$ obtained by applying Hamilton-Adams algorithm to the green channel and bilinear interpolation for both 


\begin{tabular}{|c|c|c|c|}
\hline \multicolumn{3}{|c|}{ Salt-and-Pepper noise with $d=0.3$ (TOP), $d=0.4$ (MIDDLE), $d=0.5$ (BOTTOM) } \\
\hline data $f$ & MSH $^{1}$ & MSTV & TV \\
\hline preprocessed $\bar{g}$ & NL/MSH $^{1}$ & NL/MSTV & NL/TV \\
\hline
\end{tabular}
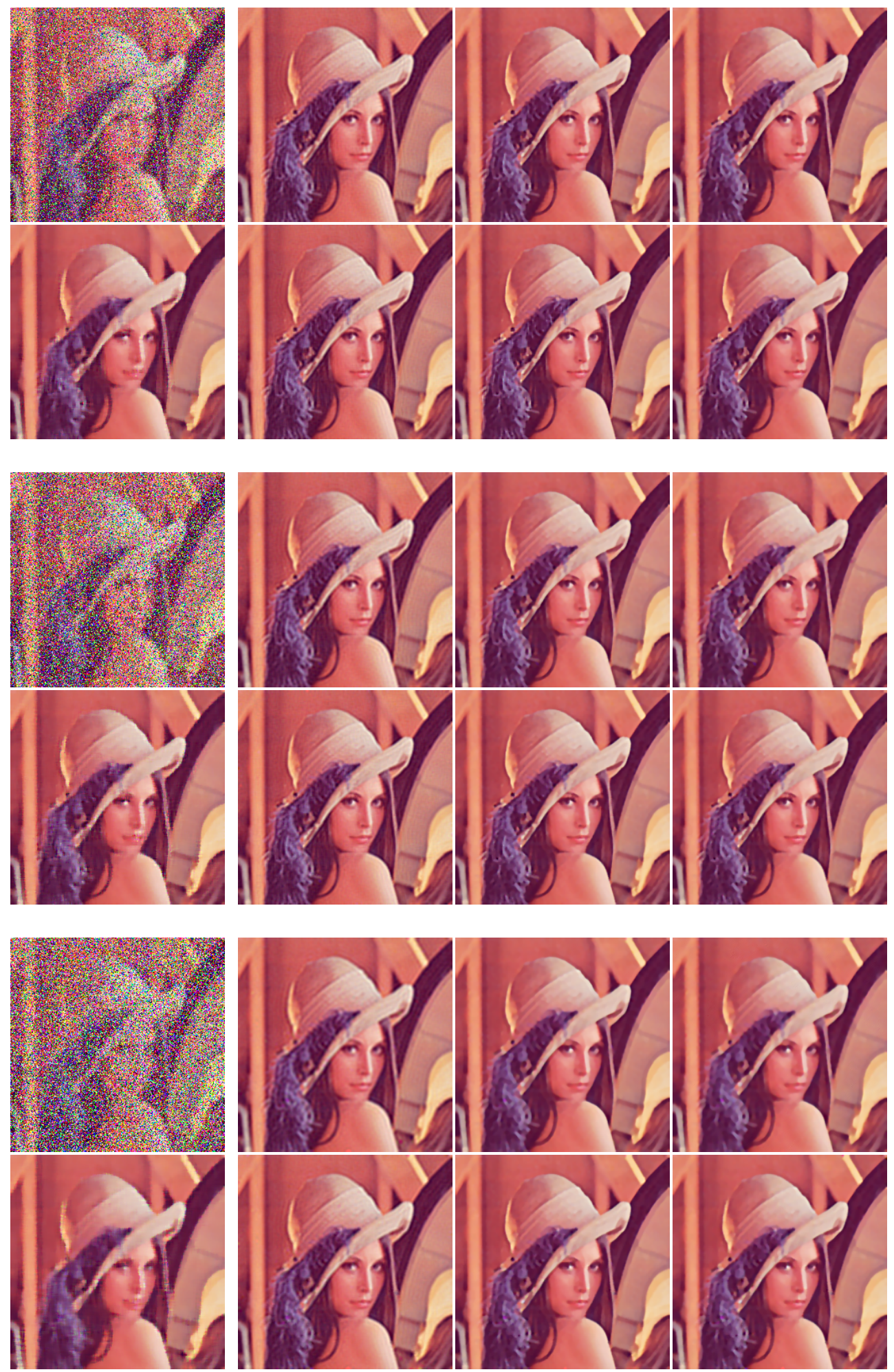

Fig. 7. Deblurring in the PReSence of SAlt-And-PepPer noise using local (MSH ${ }^{1}$, MSTV, TV) and nonlocal regularizers (NL/MSH ${ }^{1}$, NL/MSTV, NL/TV). Data $f$ : Gaussian blur kernel with $\sigma_{b}=2$, noise density $d=0.3$ (Top two rows), $d=0.4$ (Middle two rows), $d=0.5$ (Bottom two rows). 


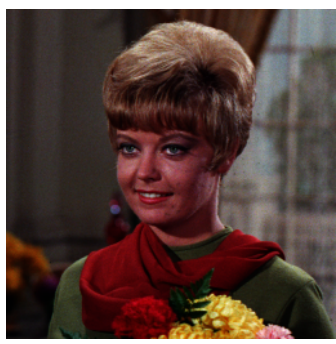

original

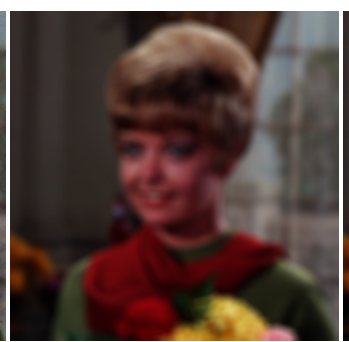

blurry $(A)$

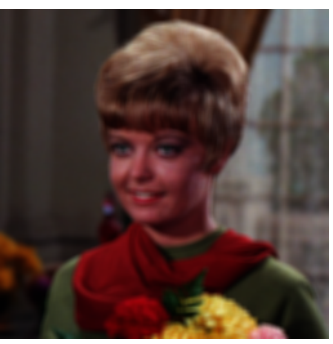

blurry (B)

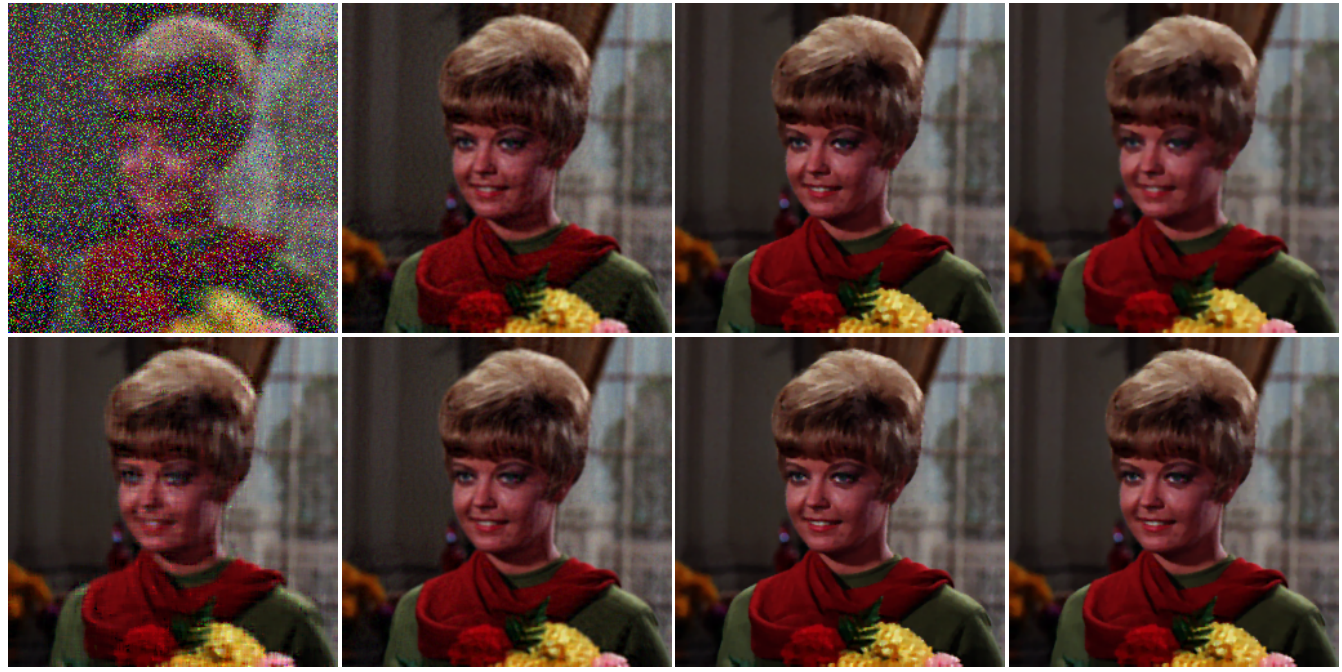

\begin{tabular}{|c|c|c|c|}
\hline$f(\mathrm{~A}): 12.4583$ & $\mathrm{MSH}^{1}: 30.9531$ & MSTV: 32.4722 & TV: 30.7566 \\
\hline $\bar{g}: 27.9720$ & $\mathrm{NL} / \mathrm{MSH}^{1}: 31.4532$ & NL/MSTV: 33.0390 & NL/TV: 32.6614 \\
\hline
\end{tabular}
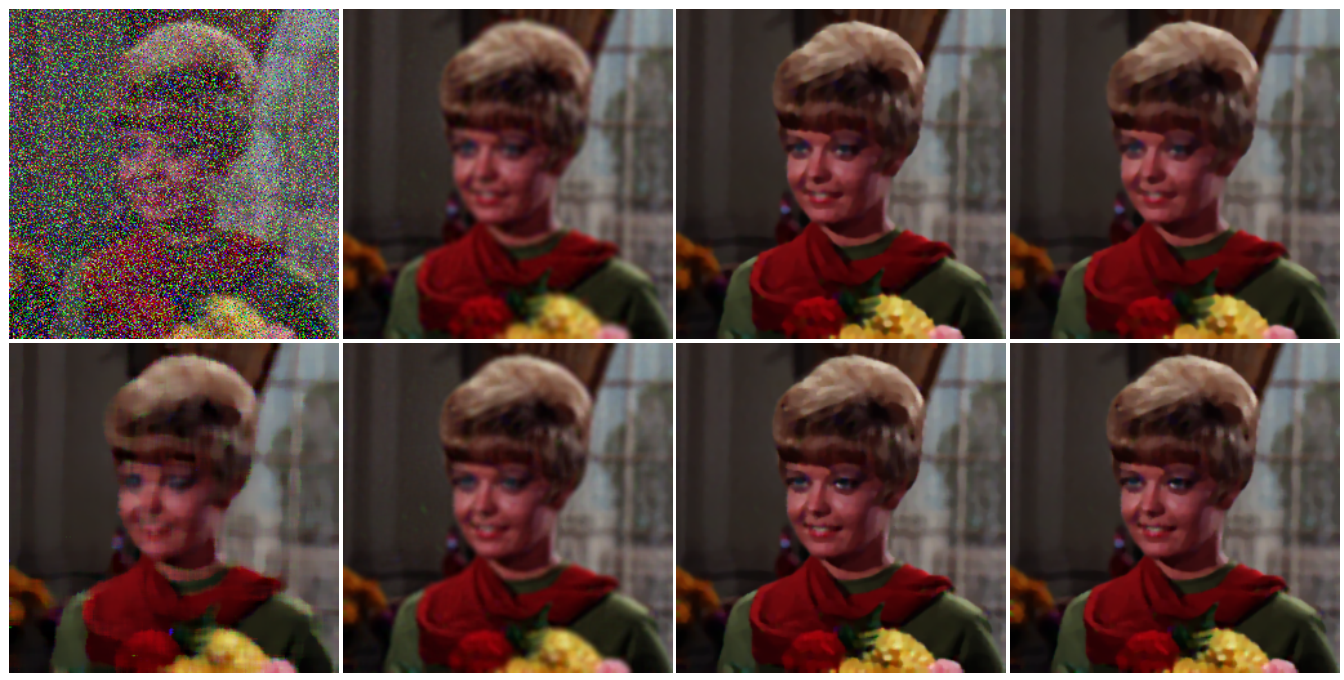

\begin{tabular}{|c|c|c|c|}
\hline$f(\mathrm{~B}): 11.2622$ & $\mathrm{MSH}^{1}: 27.6346$ & MSTV: 29.8059 & TV: 29.5530 \\
\hline $\bar{g}: 25.7044$ & $\mathrm{NL} \mathrm{MSH}^{1}: 28.1454$ & NL/MSTV: 30.1133 & NL/TV: 29.9388 \\
\hline
\end{tabular}

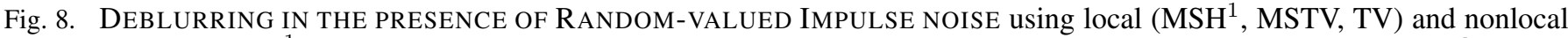
regularizers (NL/MSH ${ }^{1}$, NL/MSTV, NL/TV). (Top two rows) data $f$ : out of focus blur kernel with radius $r=5$ (A), noise density $d=0.3, \bar{g}$ with $9 \times 9$ median filter, (Bottom two rows) data $f$ : out of focus blur kernel with radius $r=3$ (B), noise density $d=0.4, \bar{g}: 11 \times 11$ median filter. 


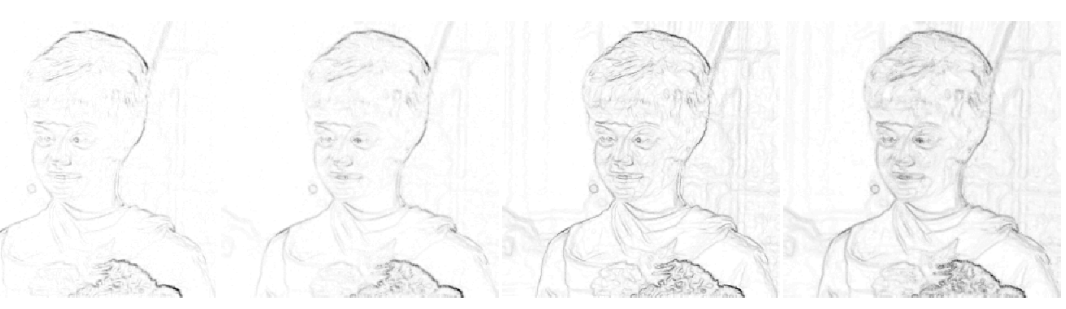

Fig. 9. Edge set $v$ obtained during the restoration process using (left to right) $\mathrm{MSH}^{1}$, NL/MSH ${ }^{1}$, MSTV, NL/MSTV in Fig. 8 Top two rows.

PARAMETERS SElEctions $(\lambda, \beta)$ FOR Fig. $2,3,7,8$

\begin{tabular}{|c|c|c|c|c|c|c|}
\hline & TV & NLTV & MSH $^{1}$ & NL/MSH $^{1}$ & MSTV & NL/MSTV \\
\hline Barbara & 17.5 & 25 & 0.4 & 0.09 & 0.065 & 0.04 \\
& 3 & 6 & 2 & 0.6 & 0.3 & 0.2 \\
Lena & 20 & 30 & 0.17 & 0.07 & 0.04 & 0.02 \\
& 13 & 23 & 0.2 & 0.08 & 0.07 & 0.04 \\
& 5 & 12 & 0.8 & 0.2 & 0.2 & 0.08 \\
Girl & 14 & 33 & 0.3 & 0.12 & 0.05 & 0.03 \\
& 2.5 & 5 & 5 & 2 & 0.4 & 0.18 \\
\hline
\end{tabular}

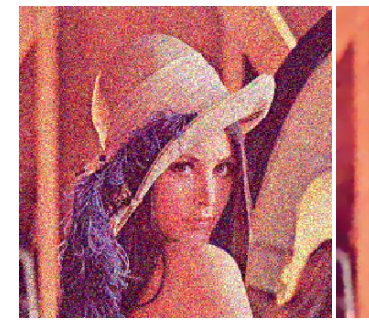

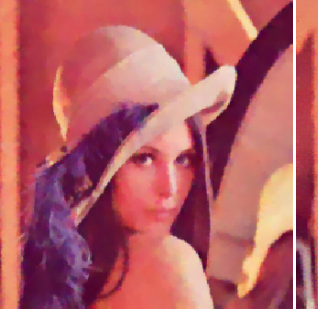

MSTV: 26.1951
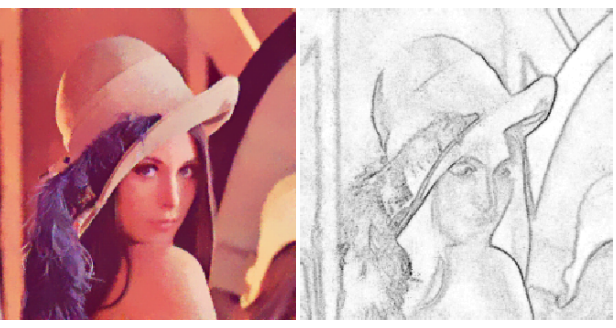

NL/MSTV: 27.1599 with edge $v$
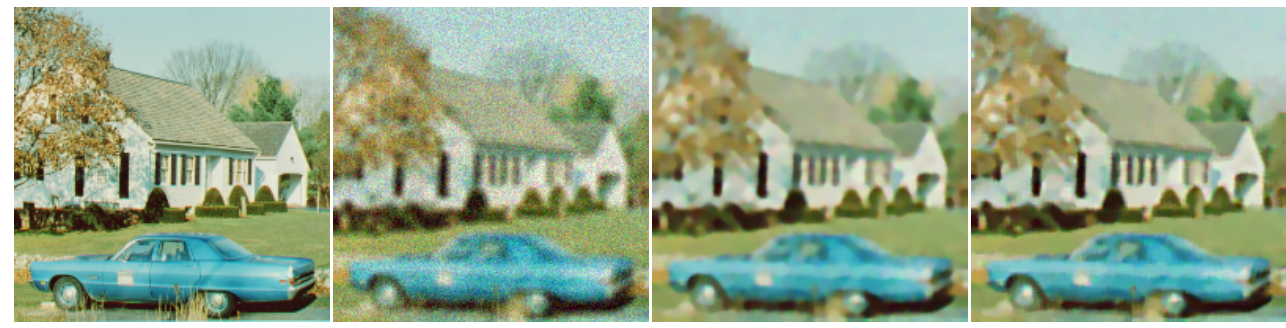

original

data $f=k * u+n$

MSTV: 22.3422

NL/MSTV: 22.7141

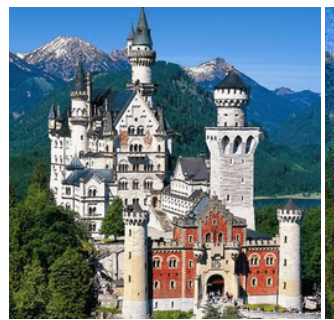

original

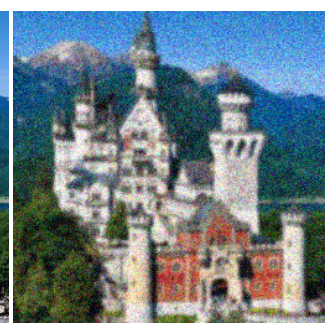

data $f=k * u+n$

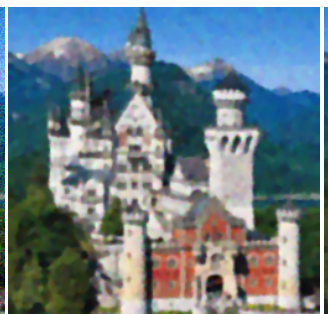

MSTV: 19.4849

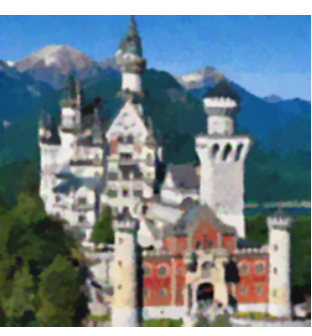

NL/MSTV: 19.8815

Fig. 10. DENOISING OR DEBluRRING IN THE PRESENCE OF GAUSSIAN NOISE. Top: noisy image $f$ with noise density $d=0.02$. Middle: out of focus blur kernel $k$ of radius $r=3$ and noise with $d=0.005$. Bottom: Gaussian blur kernel $k$ with $\sigma_{b}=1.2$ and noise with $d=0.01$. PSNR of data $f: 17.3719$ (Lena), 19.4293 (house), 17.2534 (castle). $\beta$ : (top) MSTV: 0.17, NL/MSTV: 0.07, (middle) MSTV: 0.03, NL/MSTV: 0.008, (bottom) MSTV: 0.06, NL/MSTV: 0.02. 


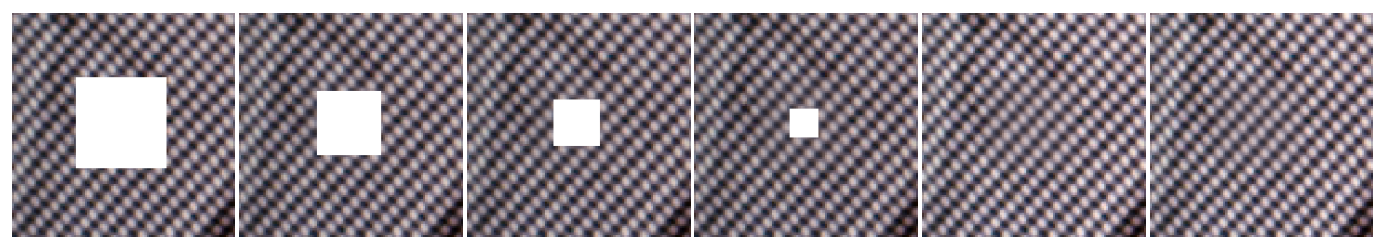
(a) data $f$
(b) 150 th
(c) 250 th
(d) 350 th
(e) $\mathrm{NL} / \mathrm{MSH}^{1}$
(f) NL/MSTV

Fig. 11. INPAINTING of $100 \times 100$ size image with $40 \times 40$ missing part. (a) data $f$, (b)-(d) process of inpainting with NL/MSH $^{1}$, recovered images using (e) $\mathrm{NL} \mathrm{MSH}^{1}$ : PSNR=35.6704, (f) $\mathbf{N L / M S T V : ~ P S N R = 3 5 . 8 0 2 4}$, with $41 \times 41$ search window, $9 \times 9$ patch.

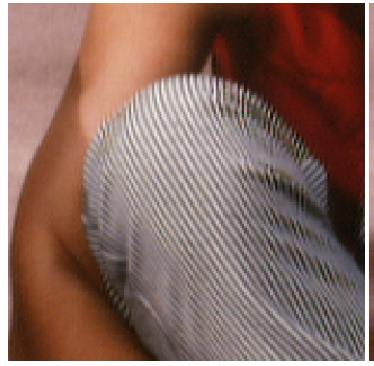

original

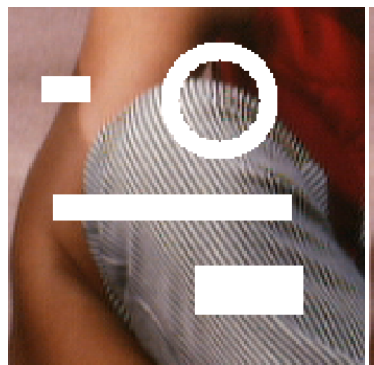

data $f$

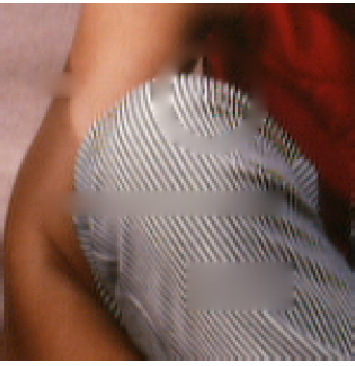

(a) $\mathrm{MSH}^{1}$

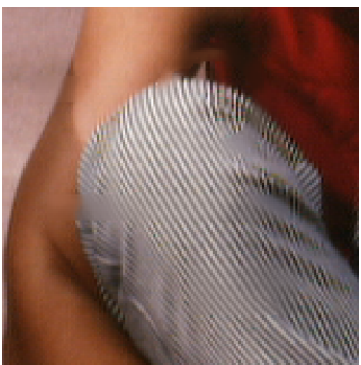

(c) $\mathrm{NL} / \mathrm{MSH}^{1}$

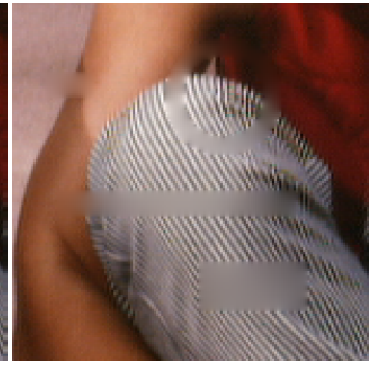

(b) MSTV

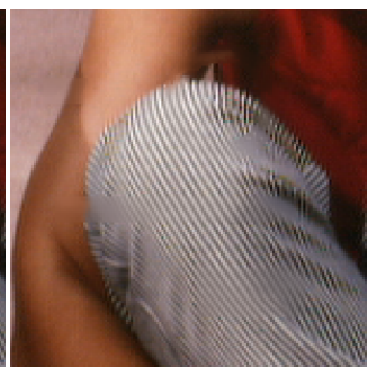

(d) NL/MSTV

Fig. 12. INPAINTING of $150 \times 150$ size image. (a)-(d): recovered images using (a) $\mathrm{MSH}^{1}$ : PSNR=29.2797, (b) MSTV: 29.4205, (c) NL/MSH ${ }^{1}$ : 34.4953, (d) NL/MSTV: 34.2406. (b), (d): $51 \times 51$ search window, $9 \times 9$ patch.

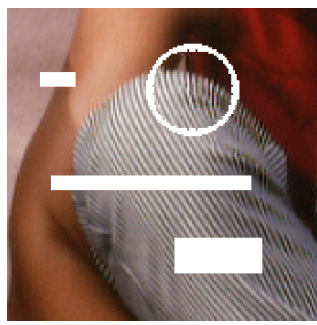

(a) 50 th

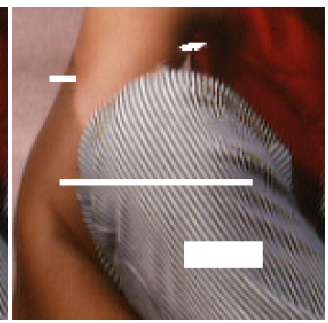

(b) 100th

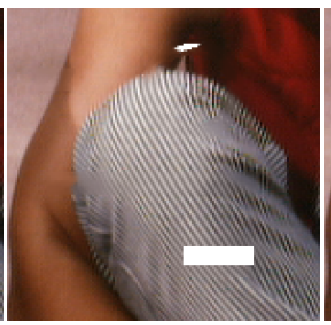

(c) 200 th

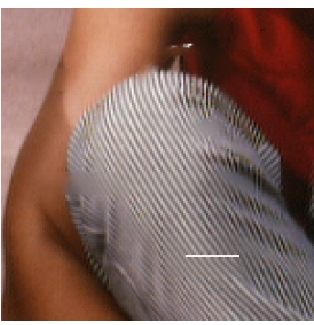

(d) 350 th

Fig. 13. The process of inpainting with $\mathrm{NL} / \mathrm{MSH}^{1}$ in 50th, 100th, 200th, 350th iterations.

$r-g$ and $b-g$. Figures 16-18 show that, with both NL/MS regularizers, the color artifacts of $u_{0}$ are much reduced, even after one iteration, and these are gradually further reduced by using decreasing sequences $h=\{16,8,4\}$ or $h=\{16,8,2\}$. Moreover, NL/MSTV produces better reconstructed images than NL/MSH ${ }^{1}$ with less color artifacts in the final images as well as higher PSNR values. Even in the case with the well-interpolated initial $u_{0}$ as in Fig. 19 , NL/MS regularizer improves $u_{0}$ in one iteration, by recovering the window parts, which can be seen in the residual with the original image. In Fig. 17, we display the reconstructed images and the PSNR values for different decreasing values of $h=\{32,16,8,4\}$, and we obtain visually similar final reconstructed image with the one of $h=\{16,8,4\}$ in Fig. 16 while the PSNR value of the final image is smaller than the one in Fig. 16. We also 
present the reconstructed images using local MS regularizers in Fig. 20, that are slightly improved from the initial $u_{0}$, but in the case where the artifacts in $u_{0}$ are severe such as the Fence and the Roof images they do not look different from the initial $u_{0}$.

Finally, we note that the parameters $\alpha, \beta$ and $\epsilon$ were selected manually to provide the best PSNR results. The smoothness parameter $\beta$ increases with the noise level, while the other parameters $\alpha, \epsilon$ are approximately fixed, $\alpha=0.001, \epsilon=0.00000001$ for deblurring-denoising, inpainting, super-resolution, demosaicing, and $\alpha=0.1$, $\epsilon=0.001$ for denoising (although in theory $\epsilon \rightarrow 0$, it is common in practice to work with a small fixed $\epsilon$ ). For the weights $w$, we use $11 \times 11$ search window with $5 \times 5$ patch for the deblurring-denoising model (or denoising), $21 \times 21$ search window with $9 \times 9$ patch for super-resolution, and $15 \times 15$ search window with $3 \times 3$ patch for demosaicing, while larger search windows are needed for inpainting. For the computational time, it takes about 5 minutes for constructing the weight function of a $256 \times 256$ image with the $11 \times 11$ search window and $5 \times 5$ patch in MATLAB on a dual core laptop with $2 \mathrm{GHz}$ processor and $2 \mathrm{~GB}$ memory. The minimization for the (local or nonlocal) MS regularizers in the deblurring-denoising model takes about 150 seconds for the computations of both $u$ using an explicit scheme based on the gradient descent method and $v$ using a semi-implicit scheme with the total iterations $5 \times(2+100)$ (without including the computation of the weight function $w(x, y)$ ). For the inpainting model with $150 \times 150$ size image, it takes about 20 minutes with total iteration numbers $5 \times(2+100)$ since we update the weight function at every 50th iteration for $u$. For both super-resolution and demosaicing, $10 \times(2+200)$ iteration numbers are needed for all regularizers.

\section{Summary AND CONCLUSIONS}

The proposed nonlocal MS regularizers, $\mathrm{NL} / \mathrm{MSH}^{1}$ and NL/MSTV, outperform the local ones on all the applications. For deblurring in the presence of impulse noise, the nonlocal regularizers (including NL/TV) incorporating an efficient preprocessing step perform very well and provide better recovered images than local ones; $\mathrm{NL} \mathrm{MSH}^{1}$ reduces ringing artifacts appeared in $\mathrm{MSH}^{1}$, and both NL/MSTV and NL/TV reduce staircase effects appeared on the local ones, resulting in more realistic images, and recover details better. For denoising or deblurring in the presence of Gaussian noise, NL/MSTV recovers fine scales better, and gives cleaner and shaper images than local regularizers as well as $\mathrm{NL} / \mathrm{MSH}^{1}$. Even for super-resolution, NL/MSTV provides the cleanest and sharpest results, and for color filter array demosaicing, NL/MSTV reconstructs images best. For inpainting, both NL/MSH ${ }^{1}$ and NL/MSTV provide superior results than the local ones by recovering textures and large missing regions. To sum up, in all the experiments (except inpainting), NL/MSTV produces superior results than local regularizers as well as $\mathrm{NL} / \mathrm{MSH}^{1}$. Moreover, NL/MSTV provides edge sets concurrently obtained with the restoration process, which is another stronger point than NL/TV, while NL/TV is computationally faster.

\section{APPENDIX I}

We show that the following regularizing functionals are semi-norms, a necessary step in the proofs of Propositions 1 and 2:

$$
\begin{aligned}
& |u|_{N L T V}=\int_{\Omega} \sqrt{\sum_{i} \int_{\Omega}\left(u^{i}(y)-u^{i}(x)\right)^{2} w(x, y) d y} d x \\
& |u|_{N L / M S T V, v}=\int_{\Omega} v^{2}(x)\left(\sqrt{\sum_{i} \int_{\Omega}\left(u^{i}(y)-u^{i}(x)\right)^{2} w(x, y) d y}\right) d x \\
& |u|_{N L / M S H^{1}, v}=\sqrt{\int_{\Omega} v^{2}(x)\left(\sum_{i} \int_{\Omega}\left(u^{i}(y)-u^{i}(x)\right)^{2} w(x, y) d y\right) d x}
\end{aligned}
$$

with $u: \Omega \rightarrow \mathbb{R}^{3}, v: \Omega \rightarrow \mathbb{R}$ and $w: \Omega \times \Omega \rightarrow \mathbb{R}$ is nonnegative and symmetric. We only need to show that these functionals satisfy the triangle inequality. 
Define $|u|=\int_{\Omega} g(x)\left(\sqrt{\sum_{i} \int_{\Omega}\left(u^{i}(y)-u^{i}(x)\right)^{2} w(x, y) d y}\right) d x$ for any nonnegative function $g: \Omega \rightarrow \mathbb{R}$, and show that $|u+v| \leq|u|+|v|$ for $u, v: \Omega \rightarrow \mathbb{R}^{3}$. First, we have the equality

$$
\begin{aligned}
& \sum_{i} \int_{\Omega}\left(\left(u^{i}+v^{i}\right)(y)-\left(u^{i}+v^{i}\right)(x)\right)^{2} w(x, y) d y \\
& =\sum_{i} \int_{\Omega}\left(u^{i}(y)-u^{i}(x)\right)^{2} w(x, y) d y+\sum_{i} \int_{\Omega}\left(v^{i}(y)-v^{i}(x)\right)^{2} w(x, y) d y \\
& +2 \sum_{i} \int_{\Omega}\left(u^{i}(y)-u^{i}(x)\right)\left(v^{i}(y)-v^{i}(x)\right) w(x, y) d y .
\end{aligned}
$$

Using Cauchy-Schwarz inequality, we have

$$
\begin{aligned}
& \int_{\Omega}\left(u^{i}(y)-u^{i}(x)\right)\left(v^{i}(y)-v^{i}(x)\right) w(x, y) d y \\
& \leq\left(\int_{\Omega}\left(\left(u^{i}(y)-u^{i}(x)\right) \sqrt{w(x, y)}\right)^{2} d y\right)^{1 / 2}\left(\int_{\Omega}\left(\left(v^{i}(y)-v^{i}(x)\right) \sqrt{w(x, y)}\right)^{2} d y\right)^{1 / 2} .
\end{aligned}
$$

Denote

$$
a^{i}=\int_{\Omega}\left(u^{i}(y)-u^{i}(x)\right)^{2} w(x, y) d y, \quad b^{i}=\int_{\Omega}\left(v^{i}(y)-v^{i}(x)\right)^{2} w(x, y) d y .
$$

Using the fact $\sum_{i} \sqrt{a^{i}} \sqrt{b^{i}} \leq \sqrt{\sum_{i} a^{i}} \sqrt{\sum_{i} b^{i}}$, we obtain

$$
\begin{aligned}
& \sum_{i} \int_{\Omega}\left(\left(u^{i}+v^{i}\right)(y)-\left(u^{i}+v^{i}\right)(x)\right)^{2} w(x, y) d y \\
& \leq \sum_{i}\left(a^{i}+b^{i}+2 \sqrt{a^{i}} \sqrt{b^{i}}\right)=\sum_{i} a^{i}+\sum_{i} b^{i}+2 \sum_{i} \sqrt{a^{i}} \sqrt{b^{i}} \\
& \leq \sum_{i} a^{i}+\sum_{i} b^{i}+2 \sqrt{\sum_{i} a^{i}} \sqrt{\sum_{i} b^{i}}=\left(\sqrt{\sum_{i} a^{i}}+\sqrt{\sum_{i} b^{i}}\right)^{2},
\end{aligned}
$$

which finally leads to

$$
\begin{aligned}
& \sqrt{\sum_{i} \int_{\Omega}\left(\left(u^{i}+v^{i}\right)(y)-\left(u^{i}+v^{i}\right)(x)\right)^{2} w(x, y) d y} \\
& \leq \sqrt{\sum_{i} \int_{\Omega}\left(u^{i}(y)-u^{i}(x)\right)^{2} w(x, y) d y}+\sqrt{\sum_{i} \int_{\Omega}\left(v^{i}(y)-v^{i}(x)\right)^{2} w(x, y) d y}
\end{aligned}
$$

Multiplying $g(x)$ and integrating both sides w.r.t $x$, we obtain

$$
\begin{aligned}
& \int_{\Omega} g(x) \sqrt{\sum_{i} \int_{\Omega}\left(\left(u^{i}+v^{i}\right)(y)-\left(u^{i}+v^{i}\right)(x)\right)^{2} w(x, y)} d y d x \\
& \leq \int_{\Omega} g(x) \sqrt{\sum_{i} \int_{\Omega}\left(u^{i}(y)-u^{i}(x)\right)^{2} w(x, y) d y} d x \\
& +\int_{\Omega} g(x) \sqrt{\sum_{i} \int_{\Omega}\left(v^{i}(y)-v^{i}(x)\right)^{2} w(x, y) d y} d x .
\end{aligned}
$$

Thus, $|u|$ satisfies the triangle inequality, so we conclude that $|u|$ is a semi-norm. Specifically, by taking $g(x)=1$ or $g(x)=v^{2}(x),|u|_{N L / T V}$ and $|u|_{N L / M S T V, v}$ are semi-norms. 
Similarly, we can also show that $|u|_{N L / M S H^{1}, v}$ is a semi-norm using Cauchy-Schwarz inequality and the fact $\sum_{i} \sqrt{a^{i}} \sqrt{b^{i}} \leq \sqrt{\sum_{i} a^{i}} \sqrt{\sum_{i} b^{i}}$ :

$$
\begin{aligned}
& \sum_{i} \int_{\Omega} \int_{\Omega} v^{2}(x)\left(\left(u^{i}+\varphi^{i}\right)(y)-\left(u^{i}+\varphi^{i}\right)(x)\right)^{2} w(x, y) d y d x \\
& =\sum_{i} \int_{\Omega} \int_{\Omega} v^{2}(x)\left(u^{i}(y)-u^{i}(x)\right)^{2} w(x, y) d y d x \\
& +\sum_{i} \int_{\Omega} \int_{\Omega} v^{2}(x)\left(\varphi^{i}(y)-\varphi^{i}(x)\right)^{2} w(x, y) d y d x \\
& +2 \sum_{i} \int_{\Omega} \int_{\Omega} v^{2}(x)\left(u^{i}(y)-u^{i}(x)\right)\left(\varphi^{i}(y)-\varphi^{i}(x)\right) w(x, y) d y d x .
\end{aligned}
$$

Using Cauchy-Schwarz inequality,

$$
\begin{aligned}
& \int_{\Omega} \int_{\Omega} v^{2}(x)\left(u^{i}(y)-u^{i}(x)\right)\left(\varphi^{i}(y)-\varphi^{i}(x)\right) w(x, y) d y d x \\
& \leq\left(\int_{\Omega} \int_{\Omega}\left(v(x)\left(u^{i}(y)-u^{i}(x)\right) \sqrt{w(x, y)}\right)^{2} d y d x\right)^{1 / 2} \\
& \left(\int_{\Omega} \int_{\Omega}\left(v(x)\left(\varphi^{i}(y)-\varphi^{i}(x)\right) \sqrt{w(x, y)}\right)^{2} d y d x\right)^{1 / 2},
\end{aligned}
$$

and using the fact $\sum_{i} \sqrt{a^{i}} \sqrt{b^{i}} \leq \sqrt{\sum_{i} a^{i}} \sqrt{\sum_{i} b^{i}}$, we have

$$
\begin{aligned}
& \sqrt{\sum_{i} \int_{\Omega} \int_{\Omega} v^{2}(x)\left(\left(u^{i}+\varphi^{i}\right)(y)-\left(u^{i}+\varphi^{i}\right)(x)\right)^{2} w(x, y) d y d x} \\
& \leq \sqrt{\sum_{i} \int_{\Omega} \int_{\Omega} v^{2}(x)\left(u^{i}(y)-u^{i}(x)\right)^{2} w(x, y) d y d x} \\
& +\sqrt{\sum_{i} \int_{\Omega} \int_{\Omega} v^{2}(x)\left(\varphi^{i}(y)-\varphi^{i}(x)\right)^{2} w(x, y) d y d x} .
\end{aligned}
$$

Hence, $|u|_{N L / M S H^{1}, v}$ satisfies the triangle inequality as well, so $|u|_{N L / M S H^{1}, v}$ is a semi-norm.

\section{APPENDIX II}

Proof of Proposition. 2. Let $[u, v]$ be a minimizing pair. Considering the variation of $F$ only with respect to $u$, we find that for any $\varphi \in N L / M S(\Omega)=\left\{u \in\left(L^{2}(\Omega)\right)^{3}:|u|_{N L / M S}<\infty\right\}$, we have

$$
\begin{aligned}
& \int_{\Omega} \sqrt{\sum_{i}\left(f^{i}-K u^{i}\right)^{2}+\eta^{2}} d x+\beta|u|_{N L / M S} \\
& \leq \int_{\Omega} \sqrt{\sum_{i}\left(f^{i}-K\left(u^{i}+\epsilon \varphi^{i}\right)\right)^{2}+\eta^{2}} d x+\beta|u+\epsilon \varphi|_{N L / M S} .
\end{aligned}
$$

Let

$$
g(\epsilon):=\sqrt{\sum_{i}\left(f^{i}-K\left(u^{i}+\epsilon \varphi^{i}\right)\right)^{2}+\eta^{2}} .
$$

Taylor's expansion gives $g(\epsilon)=\sqrt{\sum_{i}\left(f^{i}-K u^{i}\right)^{2}+\eta^{2}}-\epsilon \frac{(f-K u) \cdot(K \varphi)}{\sqrt{\sum_{i}\left(f^{i}-K u^{i}\right)^{2}+\eta^{2}}}+\frac{\epsilon^{2}}{2} g^{\prime \prime}\left(\epsilon_{\xi}\right)$ and hence

$$
\begin{array}{r}
\int_{\Omega} \sqrt{\sum_{i}\left(f^{i}-K\left(u^{i}+\epsilon \varphi^{i}\right)\right)^{2}+\eta^{2}} d x \leq \int_{\Omega} \sqrt{\sum_{i}\left(f^{i}-K u^{i}\right)^{2}+\eta^{2}} d x \\
-\epsilon\left\langle\frac{f-K u}{\sqrt{\sum_{i}\left(f^{i}-K u^{i}\right)^{2}+\eta^{2}}}, K \varphi\right\rangle+\frac{\epsilon^{2}}{2} \max _{x}\left|g^{\prime \prime}(x)\right| .
\end{array}
$$


Then, the first inequality implies that

$$
\epsilon\left\langle\frac{f-K u}{\sqrt{\sum_{i}\left(f^{i}-K u^{i}\right)^{2}+\eta^{2}}}, K \varphi\right\rangle \leq \epsilon \beta|\varphi|_{N L / M S}+\frac{\epsilon^{2}}{2} \max _{x}\left|g^{\prime \prime}(x)\right| .
$$

Dividing by $\epsilon>0$ and letting $\epsilon \downarrow 0_{+}$(while noticing that $\lim _{\epsilon \rightarrow 0} \frac{\epsilon^{2}}{2} \max _{x}\left|g^{\prime \prime}(x)\right|=0$ ) yields that for any $\varphi \in N L / M S(\Omega)$,

$$
\left\langle K^{*} \frac{f-K u}{\sqrt{\sum_{i}\left(f^{i}-K u^{i}\right)^{2}+\eta^{2}}}, \varphi\right\rangle \leq \beta|\varphi|_{N L / M S},
$$

thus,

$$
\left\|K^{*} \frac{f-K u}{\sqrt{\sum_{i}\left(f^{i}-K u^{i}\right)^{2}+\eta^{2}}}\right\|_{*} \leq \beta .
$$

Now let $\varphi=u$. Then, dividing by $\epsilon<0$, and letting $\epsilon \uparrow 0_{-}$, we obtain

$$
\left\langle K^{*} \frac{f-K u}{\sqrt{\sum_{i}\left(f^{i}-K u^{i}\right)^{2}+\eta^{2}}}, u\right\rangle \geq \beta|u|_{N L / M S} .
$$

Combining the last two inequalities concludes the proof.

\section{APPENDIX III}

We compute the Gâteaux derivative of $J$ with respect to a minimizing function $u: \Omega \rightarrow \mathbb{R}^{3}$, with $g: \Omega \rightarrow \mathbb{R}$ and $\phi: \mathbb{R} \rightarrow \mathbb{R}$, necessary in the computation of the Euler-Lagrange equations, where

$$
J(u)=\int_{\Omega} g(x) \phi\left(\left\|\nabla_{w} u\right\|^{2}(x)\right) d x .
$$

We assume that $u$ is a minimizer of $J$ and define $G(\epsilon)=J(u+\epsilon h)$ for $\epsilon \in \mathbb{R}$ and a test function $h$. Then

$$
G(\epsilon)=\int_{\Omega} g(x) \phi\left(\left\|\nabla_{w}(u+\epsilon h)\right\|^{2}(x)\right) d x .
$$

By differentiating $G$ w.r.t $\epsilon$, we obtain

$$
\begin{aligned}
G^{\prime}(\epsilon)= & J^{\prime}(u+\epsilon h) h \\
= & \int_{\Omega} g(x) \phi^{\prime}\left(\left\|\nabla_{w}(u+\epsilon h)\right\|^{2}(x)\right) 2 \int_{\Omega}((u(y)-u(x)) \\
& +\epsilon(h(y)-h(x)))(h(y)-h(x)) w(x, y) d y d x .
\end{aligned}
$$

Taking $\epsilon=0$, we obtain the variation of $J$ with respect to $u$ i.e.

$$
\begin{aligned}
& J^{\prime}(u) h=G^{\prime}(0) \\
= & 2 \sum_{i} \int_{\Omega} g(x) \phi^{\prime}\left(\left\|\nabla_{w}(u)\right\|^{2}(x)\right) \\
& {\left[\int_{\Omega}(u(y)-u(x))(h(y)-h(x)) w(x, y) d y\right] d x } \\
= & 2 \int_{\Omega} \int_{\Omega} g(x) \phi^{\prime}\left(\left\|\nabla_{w}(u)\right\|^{2}(x)\right)(u(y)-u(x)) w(x, y) d x h(y) d y \\
& -2 \int_{\Omega} g(x) \phi^{\prime}\left(\left\|\nabla_{w}(u)\right\|^{2}(x)\right)\left[\int_{\Omega}(u(y)-u(x)) w(x, y) d y\right] h(x) d x \\
= & 2 \int_{\Omega} \int_{\Omega} g(y) \phi^{\prime}\left(\left\|\nabla_{w}(u)\right\|^{2}(y)\right)(u(x)-u(y)) w(y, x) d y h(x) d x \\
& -2 \int_{\Omega} g(x) \phi^{\prime}\left(\left\|\nabla_{w}(u)\right\|^{2}(x)\right)\left[\int_{\Omega}(u(y)-u(x)) w(x, y) d y\right] h(x) d x \\
= & -2 \int_{\Omega} \int_{\Omega} g(y) \phi^{\prime}\left(\left\|\nabla_{w}(u)\right\|^{2}(y)\right)(u(y)-u(x)) w(x, y) d y h(x) d x \\
& -2 \int_{\Omega} g(x) \phi^{\prime}\left(\left\|\nabla_{w}(u)\right\|^{2}(x)\right)\left[\int_{\Omega}(u(y)-u(x)) w(x, y) d y\right] h(x) d x
\end{aligned}
$$


where $\phi^{\prime}(s)$ is the derivative of $\phi$ with respect to $s$ and $w(x, y)=w(y, x)$. Hence, we obtain

$$
L u=-2 \int_{\Omega}(u(y)-u(x)) w(x, y)\left[\left(g(y) \phi^{\prime}\left(\left\|\nabla_{w}(u)\right\|^{2}(y)\right)+g(x) \phi^{\prime}\left(\left\|\nabla_{w}(u)\right\|^{2}(x)\right)\right] d y\right.
$$

where the operator $L$ is the gradient flow corresponding to the functional $J$.

Specifically, by taking $g(x)=v^{2}(x)$ and $\phi(s)=s$ or $\phi(s)=\sqrt{s}$, we obtain two functionals and the corresponding gradient flows:

$$
\begin{aligned}
J^{N L / M S H^{1}}(u) & =\int_{\Omega} v^{2}(x)\left\|\nabla_{w} u\right\|^{2}(x) d x: \\
L^{N L / M S H^{1}} u & =-2 \nabla_{w} \cdot\left(v^{2}(x) \nabla_{w} u(x)\right) \\
& =-2 \int_{\Omega}(u(y)-u(x)) w(x, y)\left[v^{2}(y)+v^{2}(x)\right] d y \\
J^{N L / M S T V}(u) & =\int_{\Omega} v^{2}(x)\left\|\nabla_{w} u\right\|(x) d x: \\
L^{N L / M S T V} u & =-\nabla_{w} \cdot\left(v^{2}(x) \frac{\nabla_{w} u(x)}{\left\|\nabla_{w} u(x)\right\|}\right) \\
& =-\int_{\Omega}(u(y)-u(x)) w(x, y)\left[\frac{v^{2}(y)}{\left\|\nabla_{w} u\right\|(y)}+\frac{v^{2}(x)}{\left\|\nabla_{w} u\right\|(x)}\right] .
\end{aligned}
$$

\section{REFERENCES}

[1] Alicandro, R., Braides, A., Shah, J.: Free-discontinuity problems via functionals involving the $L^{1}$-norm of the gradient and their approximation. Interfaces Free Bound. 1, 17-37 (1999)

[2] Aubert, G., Kornprobst, P.: Mathematical Problems in Image Processing: Partial Differential Equations and the Calculus of Variations. Applied Mathematical Sciences, vol. 147. Springer, Heidelberg (2001)

[3] Hamilton, J.F., Adams, J.E.: Adaptive color plan interpolation in single sensor color electronic camera. U.S. Patent 5 629 734, (1997)

[4] Aujol, J.F., Ladjal, S., Masnou, S.: Exemplar-Based Inpainting from a Variational Point of View. (2009)

[5] Alliney, S.: Digital Filters as Absolute Norm Regularizers. IEEE TSP 40(6): 1548-1562 (1992)

[6] Ambrosio, L., Tortorelli, V.M.: On the approximation of free discontinuity problems. BUMI 6-B: 105-123 (1992)

[7] Bar, L., Sochen, N., Kiryati, N.: Semi-Blind Image Restoration via Mumford-Shah Regularization. IEEE TIP 15(2), 483-493 (2006)

[8] Bar, L., Sochen, N., Kiryati, N.: Image deblurring in the presence of impulsive noise. IJCV 70: 279-298 (2006)

[9] Bar, L., Brook, A., Sochen, N., Kiryati, N.: Deblurring of Color Images Corrupted by Impulsive Noise. IEEE TIP 16(4): 1101-1111 (2007)

[10] Bayer, B.E.: Color imaging array, U.S. Patent 3971 065, (1976)

[11] Bertalmío, M., Sapiro, G., Caselles, V., Ballester, C.: Image inpainting. In: Siggraph 2000, pp. 417-424 (2000)

[12] Bertalmio, M., Vese, L.A., Sapiro, G., Osher, S.: Simultaneous Structure and Texture Image Inpainting. IEEE TIP 12(8) (2003)

[13] Bertalmio, M., Bertozzi, A.L., Sapiro, G.: Navier-Stokes, fluid dynamics, and image and video inpainting, in: Proc. IEEE Computer Vision and Pattern Recognition, (2001)

[14] Blomgren, P., Chan, T.F.: Color TV: Total variation methods for restoration of vector-valued images. IEEE TIP 7(3): 304-309 (1998)

[15] Bregman, L.M. The relaxation method for finding common points of convex sets and its application to the solution of problems in convex programming. USSR Computational Mathematics and Mathematical Physics 7: 200-217 (1967)

[16] Bresson, X., Chan, T.F.: Non-local unsupervised variational image segmentation models. UCLA C.A.M. Report 08-67 (2008)

[17] Bresson, X., Chan, T.F.: Fast Minimization of the Vectorial Total Variation Norm and Applications to Color Image Processing. Inverse Problems and Imaging, 2(4): 455-484 (2008)

[18] Brook, A., Kimmel, R., Sochen, N.: Variational restoration and edge detection for color images. JMIV 18: 247-268 (2003)

[19] Buades, A., Coll, B., Morel, J.M.: A non-local algorithm for image denoising, IEEE Int. Conf. on Computer Vision and Pattern Recognition, (2005)

[20] Buades, A., Coll, B., Morel, J.M.: A review of image denoising algorithms, with a new one. SIAM MMS 4(2): 490-530 (2005)

[21] Buades, A., Coll, B., Morel, J.M.: Image enhancement by non-local reverse heat equation. Preprint CMLA 2006-22 (2006)

[22] Buades, A., Coll, B., Morel, J.M., Sbert, C.: Non local demosaicing. CMLA Preprint 2007-15 (2007)

[23] Caselles, V., Sapiro, G., Ballester, C., Bertalmio, M., Verdera, J.: Filling-in by joint interpolation of vector fields and grey levels, IEEE Trans. Image Process. 10:1200-1211 (2001)

[24] Caselles, V., Bertalmio, M., Sapiro, G., Ballester, C.: Image inpainting, in: Comput. Graph. (SIGGRAPH 2000), July 2000, pp. 417-424

[25] Chan, T.F., Kang, S.H., Shen, J.: Euler's Elastica and Curvature Based inpainting SIAM J. Appl. Math., 63(2): 564-592 (2002)

[26] Chan, T.F., Shen, J.: Local inpainting models and TV inpainting, SIAM J. Appl. Math. 62:1019-1043 (2001)

[27] Chan, T.F., Wong, C.-K.: Total Variation Blind Deconvolution. IEEE Transactions on Image Processing, 7(3):370-375 (1998)

[28] Coifman, R.R., Lafon, S., Lee, A.B., Maggioni, M., Nadler, B., Warner, F., Zucker, S.W.: Geometric diffusions as a tool for harmonic analysis and structure definition of data: Diffusion maps. Proc. of the Nat. Ac. of Sciences 102, 7426-7431 (2005) 
[29] Criminisi, A., Pérez, P., Toyama, K.: Region filling and object removal by exemplar based image inpainting. IEEE Trans. on Image Processing 13, 1200-1212 (2004)

[30] Datsenko, D., Elad, M.: Example-based single image super-resolution: A global map approach with outlier rejection. Journal of Mult. System and Sig. Proc. 18, 103-121 (2007)

[31] Demanet, L., Song, B., Chan, T.: Image inpainting by correspondence maps: a deterministic approach. In Proc. VLSM, Nice, (2003)

[32] Effros, A., Leung, T.: Texture synthesis by non-parametric sampling. ICCV (1999)

[33] Elad, M., Starck, J.L., Donoho, D., Querre, P.: Simultaneous cartoon and texture image inpainting using morphological component analysis (MCA). Journal on Applied and Computational Harmonic Analysis 19, 340-358 (2005)

[34] Elmoataz, A., Lezoray, O., Bougleux, S.: Nonlocal discrete regularization on weighted graphs: a framework for image and manifold processing. IEEE Tr. on Image Processing 17(7), 1047-1060 (2008)

[35] Esedoglu, S., Shen, J.: Digital inpainting based on the Mumford-Shah-Euler image model. European J. Appl. Math., 13:353-370, (2002)

[36] Farsiu, S., Robinson, D., Elad, M., Milanfar, P.: Advances and challenges in superresolution. Int. Journal of Imaging Sys. and Tech. 14, 47-57 (2004)

[37] Freeman, W.T., Jones, T.R., Pasztor, E.C.: Example-based super-resolution. IEEE Computer Graphics and Applications 22, 56-65 (2002)

[38] Geman, D., Reynolds, G.: Constrained Restoration and the Recovery of Discontinuities, IEEE TPAMI 14(3), 367-383 (1992)

[39] Gilboa, G., Darbon, J., Osher, S., Chan, T.: Nonlocal convex functionals for image regularization. UCLA CAM Report 06-57 (2006)

[40] Gilboa, G., Osher, S.: Nonlocal linear image regularization and supervised segmentation. SIAM MMS 6(2): 595-630 (2007)

[41] Gilboa, G., Osher, S.: Nonlocal operators with applications to image processing. SIAM MMS 7(3): 1005-1028 (2008)

[42] Gunturk, B.K., Altunbasak, Y., Mersereau,R.M.: Color plane interpolation using alternating projections, IEEE Trans. on Image Processing, vol. 11, no. 9, pp. 997-1013, (2002)

[43] Gunturk, B.K., Glotzbach, J., Altunbasak, Y., Schafer, R.W., Mersereau, R.M.: Demosaicing: color filter array interpolation, IEEE Signal processing magazine, Vol. 44, (2005)

[44] He, L., Marquina, A., Osher, S.: Blind Deconvolution Using TV Regularization and Bregman Iteration. International Journal of Imaging Systems and Technology, 15:74-83, (2005)

[45] Hirakawa, K., Paks, T.W.: Adaptive homogeneity-directed demosaicing algorithm, IEEE Trans. on Image Processing, Vol. 14, no. 3, pp. 360-369, (2005)

[46] Jung, M., Vese, L.A.: Nonlocal variational image deblurring models in the presence of Gaussian or impulse noise. LNCS 5567: 402-413 (2009)

[47] Jung, M., Bresson, X., Chan, T.F., Vese, L.A.: Color Image Restoration Using Nonlocal Mumford-Shah Regularizers. LNCS 5681: 373-387 (2009)

[48] Kimmel, R.: Demosaicing: image reconstruction from color CCD samples, IEEE Trans. on Image Processing, Vol. 8, no. 9, pp. 1221-1228, (1999)

[49] Kindermann, S., Osher, S., Jones, P.W.: Deblurring and denoising of images by nonlocal functionals. SIAM MMS 4(4): 1091-1115 (2005)

[50] Lou, Y., Zhang, X., Osher, S., Bertozzi, A.: Image recovery via nonlocal operators. UCLA C.A.M. Report 08-35 (2008)

[51] Lu, W., Tan, Y.-P.: Color Filter Array Demosaicing: New Method and Performance Measures, IEEE Trans. on Image Processing, Vol 12, no. 10, pp. 1194-1210, (2003)

[52] Mahmoudi, M., Sapiro, G.: Fast Image and Video Denoising via Non-Local Means of Similar Neighborhoods, IEEE Signal Processing Letters, vol. 12 (12), (2005).

[53] Malgouyres, F., Guichard, F: Edge direction preserving image zooming: a mathematical and numerical analysis. SIAM NA 39(1): 1-37 (2001)

[54] Masnou, S. and Morel, J.M.: Level Lines-Based Disocclusion. IEEE Int. Conf. Image Processing, Chicago, vol. 3, pp. 259-263 (1998)

[55] Masnou, S. and Morel, J.M.: Disocclusion: a variational approach using level lines. IEEE Trans. Image Process, 11, 68-76 (2002)

[56] Meyer, Y.: Oscillating Patterns in Image Processing and Nonlinear Evolution Equations. Univ. Lecture Ser. 22., Am. Math. Soc., Providence (2002)

[57] Molina, R., Mateos, J., Katsaggelos, A., Vega, M.: Bayesian multichannel image restoration using compound Gauss-Markov random fields. IEEE Trans. Image Process., vol. 12, no. 12, pp. 1642-1654, Dec. (2003)

[58] Mumford, D., Shah, J.: Optimal approximations by piecewise smooth functions and associated variational problems. CPAM 42: 577-685 (1989).

[59] Nikolova, M.: Minimizers of cost-functions involving non-smooth data-fidelity terms. Application to the processing of outliers. SIAM NA 40(3): 965-994 (2002)

[60] Osher, S., Burger, M., Goldfarb, D., Xu, J., Yin, W.: An iterative regularization method for total variation based image restoration. SIAM MMS 4: 460-489 (2005)

[61] Pérez, P., Gangnet, M., Blake, A.: PatchWorks: Example-based region tiling for image editing. Research Report Microsoft Research, MSR-TR-2004-04, (2004)

[62] Peyré, G.: Image processing with non-local spectral bases. SIAM Multiscale Modeling and Simulation Vol. 7(2), p.703-730 (2008)

[63] Peyré, G., Bougleux, S., Cohen, L.: Non-local regularization of inverse problems. ECCV, Part III, LNCS 5304: 57-68 (2008)

[64] Protter, M., Elad, M., Takeda, H., Milanfar, P.: Generalizing the Non-Local-Means to super-resolution reconstruction. IEEE TIP 18(1): 36-51 (2009)

[65] Rudin, L., Osher, S., Fatemi, E.: Nonlinear total variation based noise removal algorithms. Phys. D 60, 227-238 (1996)

[66] Rudin, L., Osher, S.: Total variation based image restoration with free local constraints. IEEE ICIP 1, 31-35, (1994)

[67] Shah, J.: A common framework for curve evolution, segmentation and anisotropic diffusion. IEEE CVPR, 136-142 (1996)

[68] Shen, J., Chan, T.F.: Variational image inpainting. 58(5): 579-619, CPAM (2005)

[69] Smith, S.M., Brady, J.M.: SUSAN - a new approach to low level image processing. International Journal of Computer Vision 23, 45-78 (1997) 
[70] Starck, J.-L., Elad, M. and Donoho, D. Image decomposition via the combination of sparse representations and variational approach. IEEE Trans. Image Process, 14, 1570-1582 (2005)

[71] Szlam, A., Maggioni, M., Coifman, R.R.: A general framework for adaptive regularization based on diffusion processes on graphs. Journ. Mach. Learn. Res. 9, 1711-1739 (2008)

[72] Tadmor, E., Nezzar, S., Vese, L.: Multiscale hierarchical decomposition of images with applications to deblurring, denoising and segmentation: CMS 6(2), 281-307 (2008)

[73] Tichonov, A., Arsensin, V.: Solution of ill-posed problems. New York: Wiley (1977)

[74] Tomasi, C., Manduchi, R.: Bilateral filtering for gray and color images. In: Int. Conf. on Computer Vision, pp. 839-846. IEEE Computer Society, Los Alamitos (1998)

[75] Wei, L.Y., Levoy, M.: Fast texture synthesis using tree-structured vector quantization. In: SIGGRAPH 2000, pp. 479-488 (2000)

[76] Yaroslavsky, L.P.: Digital image processing: An Introduction. Springer (1985)

[77] Zhang, X., Burger, M., Bresson, X., Osher, S.: Bregmanized Nonlocal Regularization for Deconvolution and Sparse Reconstruction. UCLA CAM report 09-03 (2009)

[78] Zhou, D., Scholkopf, B.: A Regularization Framework for Learning from Graph Data. In: Workshop on Statistical Relational Learning and Its Connections to Other Fields. (2004)

[79] Zhou, D., Scholkopf., B.: Regularization on discrete spaces. In Pattern Recognition, Proceedings of the 27th DAGM Symposium, Berlin, Germany, pages 361-368, (2005) 

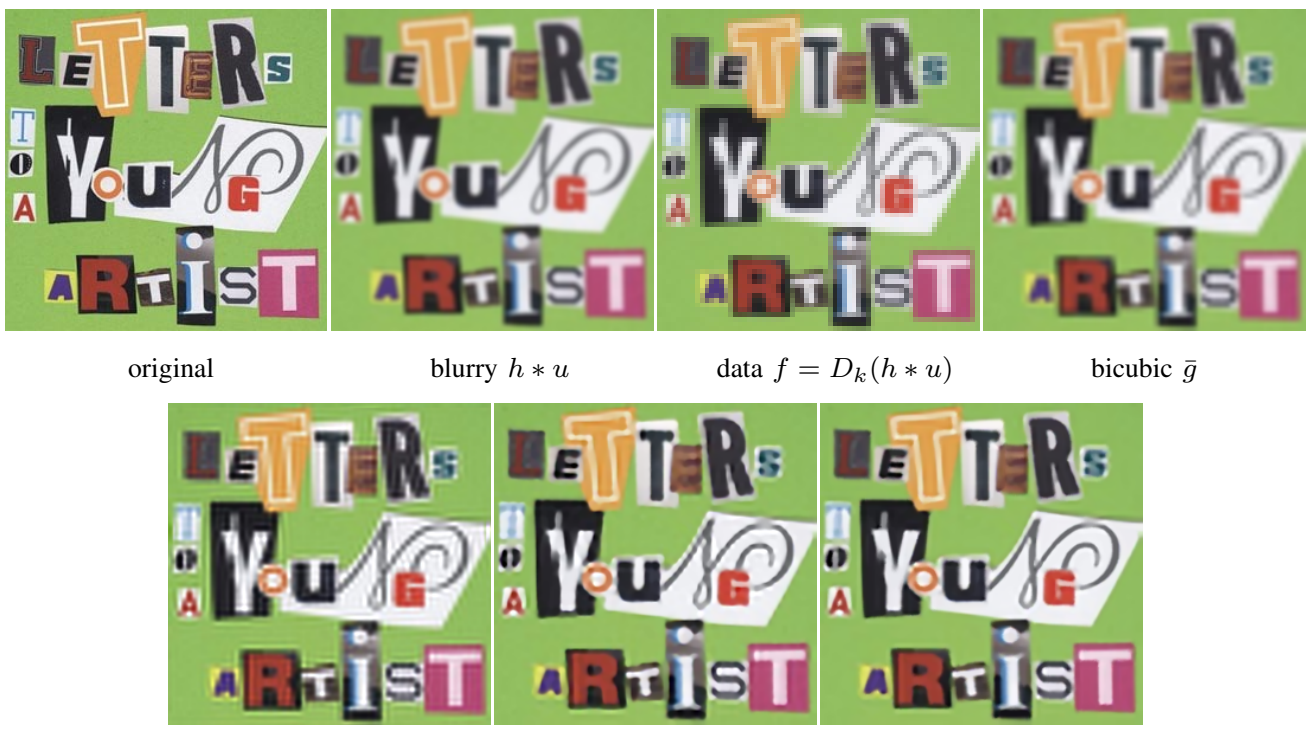

bicubic $\bar{g}+$ LR: $17.9792 \quad$ MSTV: $23.4551 \quad$ NL/MSTV: 24.3336

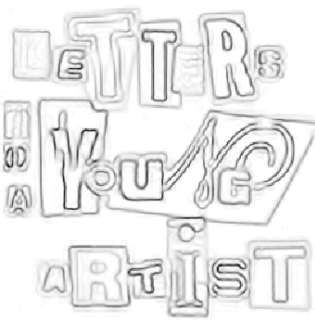

MSTV

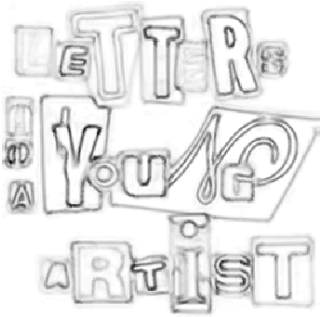

NL/MSTV

Fig. 14. SUPER-RESOLUTION of a still image. Top: original image of size $272 \times 272$, blurred image $h * u$ with Gaussian blur kernel $h$ with $\sigma_{b}=2$, down-sampled data $f=D_{k}(h * u)$ of size $68 \times 68$ with $k=4$, preprocessed (up-sampled) image $\bar{g}$ using bicubic interpolation. Middle: (left) recovered image by applying Lucy-Richarson deconvolution algorithm to $\bar{g}$ with 10 iteration number, recovered images using (middle) MSTV, (right) NL/MSTV. PSNR: $h * u: 21.9423, \bar{g}: 18.3970$.

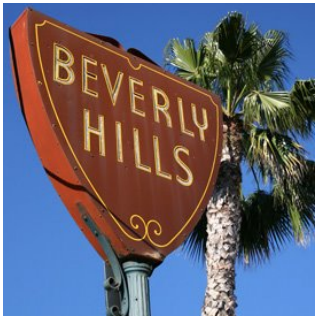

original

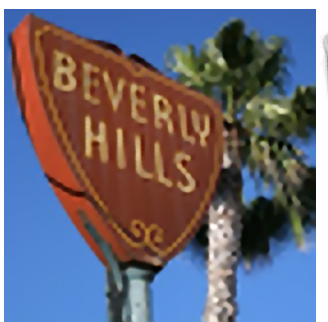

MSTV: 21.8869

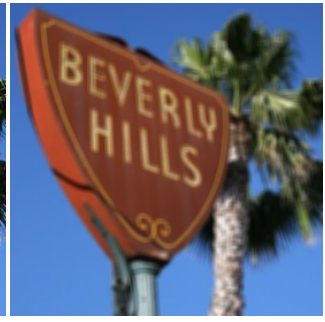

blurry $h * u$

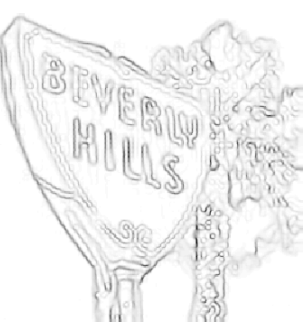

V: 21.8869

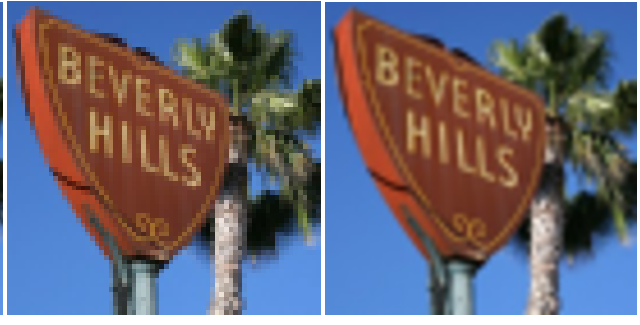

data $f=D_{k}(h * u)$

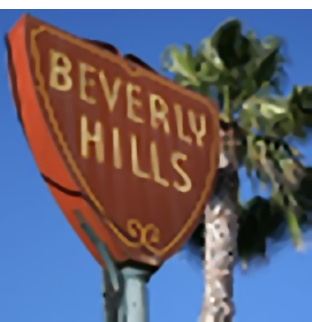

NL/MSTV: 22.1116

Fig. 15. SUPER-RESOLUTION of a still image. Top: original image of size $272 \times 272$, blurred image $h * u$ with out of focus blur kernel $h$ with radius $r=3$, down-sampled data $f=D_{k}(h * u)$ of size $68 \times 68$ with $k=4$, preprocessed (up-sampled) image $\bar{g}$ using bicubic interpolation. Bottom: recovered images using (left) MSTV, (right) NL/MSTV. PSNR: $h * u: 21.7459$, $\bar{g}: 19.9989$. 

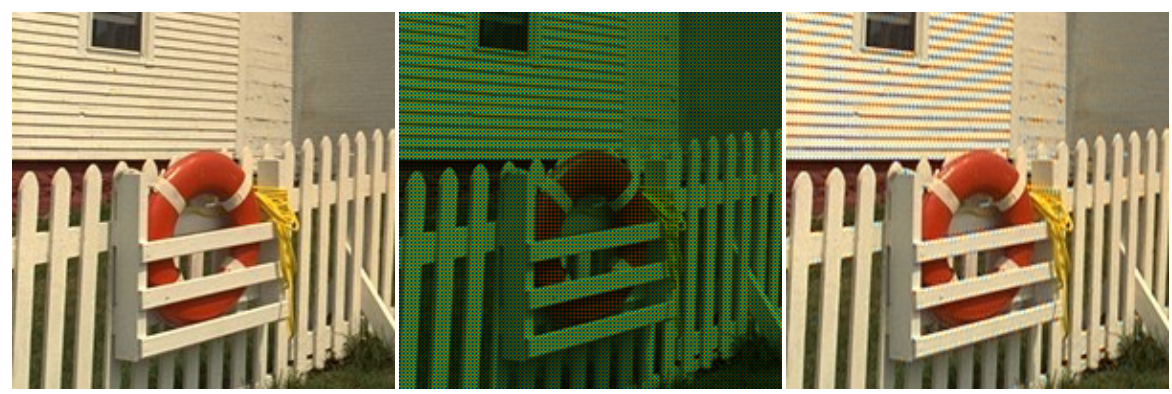

original

data $f$

Hamilton-Adams: 27.3076

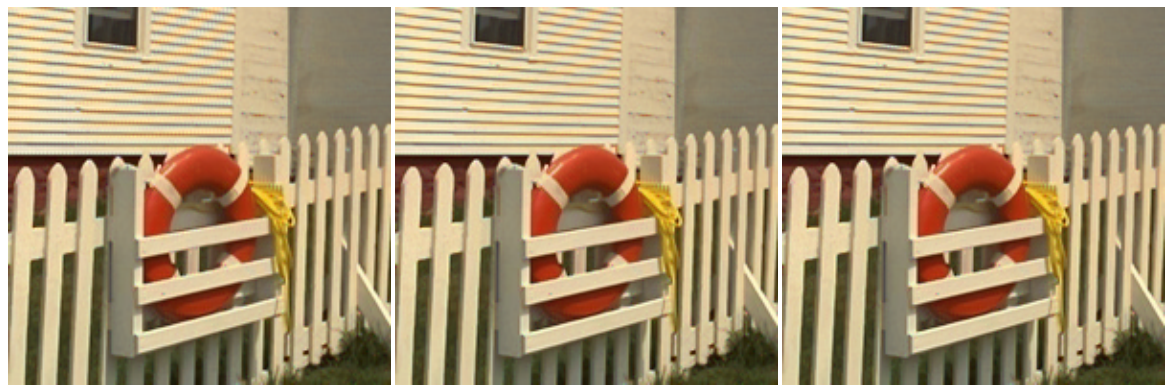

$\mathrm{NL} / \mathrm{MSH}^{1}$ with $h=\{16,8,4\}:(1 \mathrm{st}) 29.3584$, (2nd) 29.5858, (3rd) 29.5903

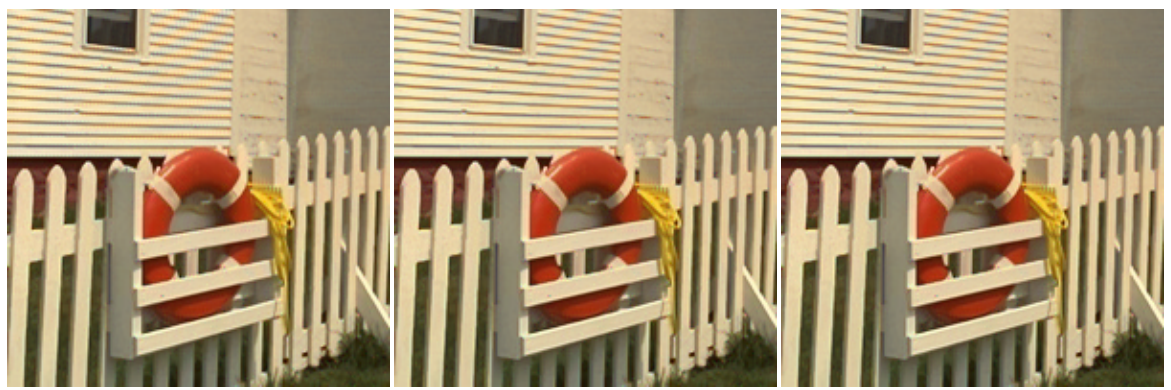

NL/MSTV with $h=\{16,8,4\}$ : (1st) 29.5101, (2nd) 29.7029, (3rd) 29.7128

Fig. 16. Demosaicing using NL/MS regularizers with iterative algorithm. Top: original image, data $f$, interpolated image using Hamilton-Adams for green and bilinear interpolation for $r-g$ and $b-g$. Middle, Bottom rows: demosaiced images using (middle) NL/MSH ${ }^{1}$ and (bottom) NL/MSTV with decreasing sequence of $h=\{16,8,4\}$ and corresponding PSNR values.

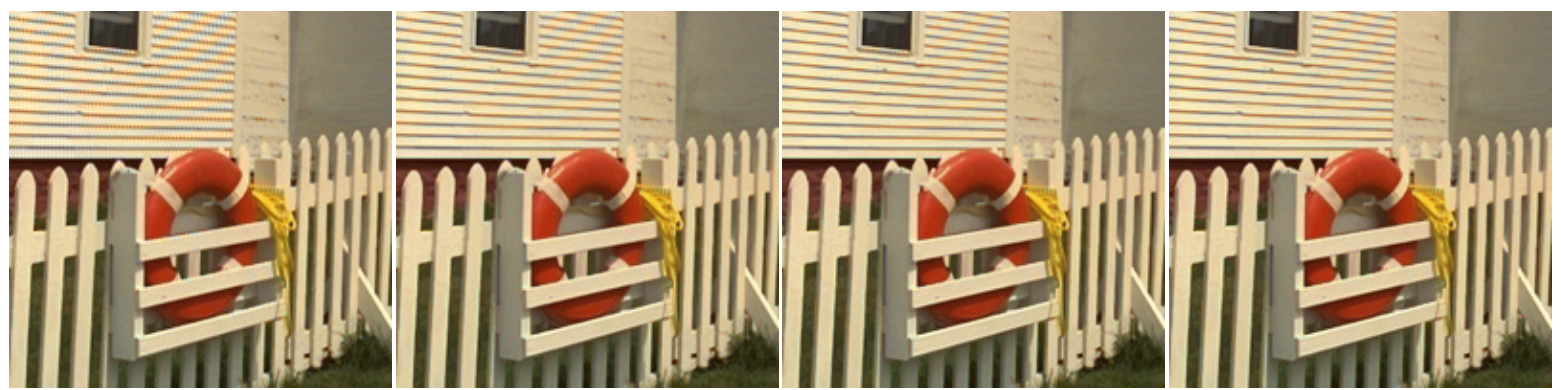

Fig. 17. Demosaiced images using NL/MSTV with different sequence of $h=\{32,16,8,4\}$. PSNR: (1st) 28.6163, (2nd) 29.0343, (3rd) 29.2111, (4th) 29.2174. Note that the PSNR values of NL/MSH ${ }^{1}$ for this case are 28.4773, 28.8928, 29.0763, 29.1242 . 


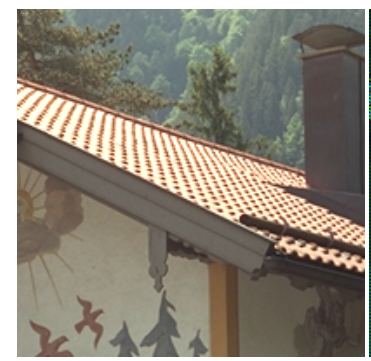

original

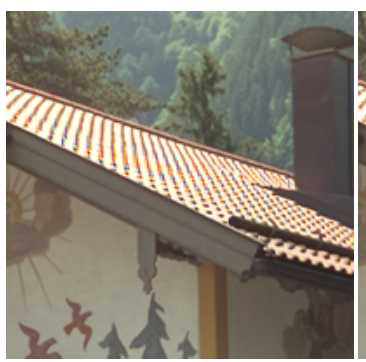

$\mathrm{NL} / \mathrm{MSH}^{1}$ with $h=\{1$

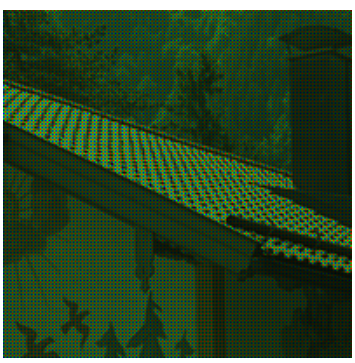

data $f$

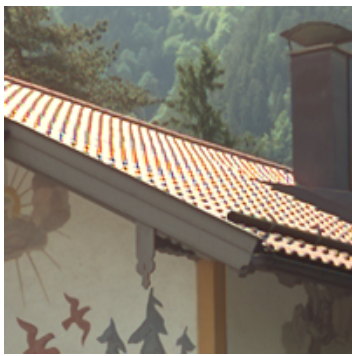

Hamilton-Adams: 26.5008
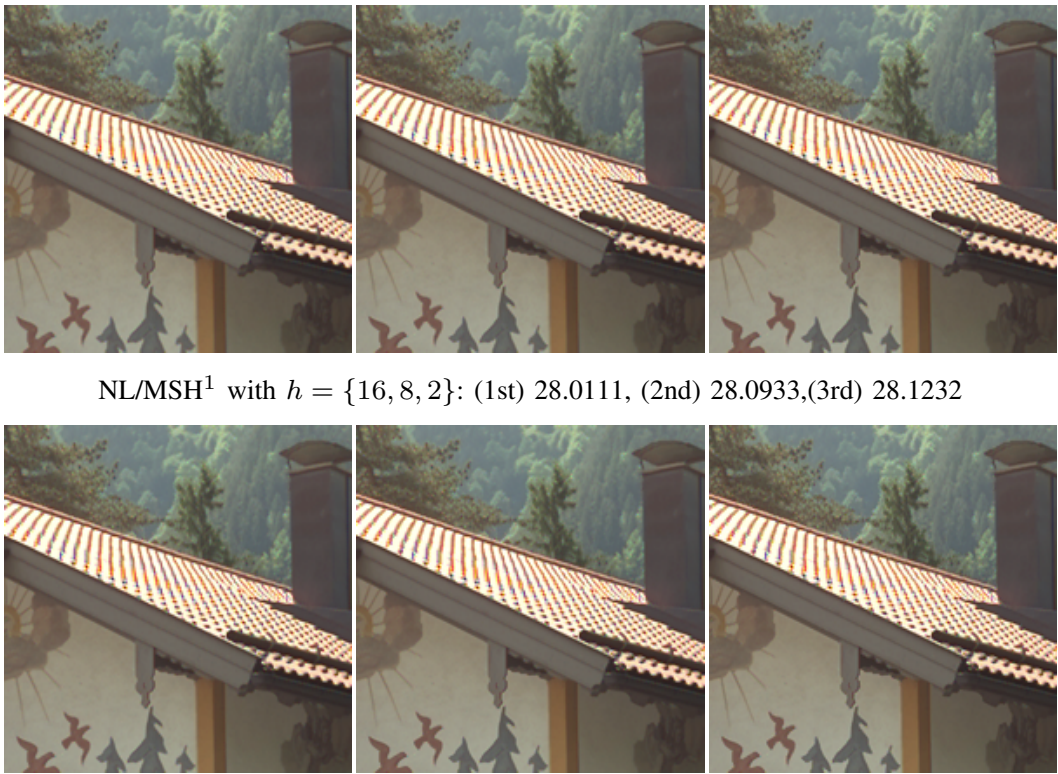

NL/MSTV with $h=\{16,8,2\}$ : (1st) 28.1480, (2nd) 28.2220, (3rd) 28.2615

Fig. 18. DemosAICING using NL/MS regularizers with $h=\{16,8,2\}$, and corresponding PSNR values.

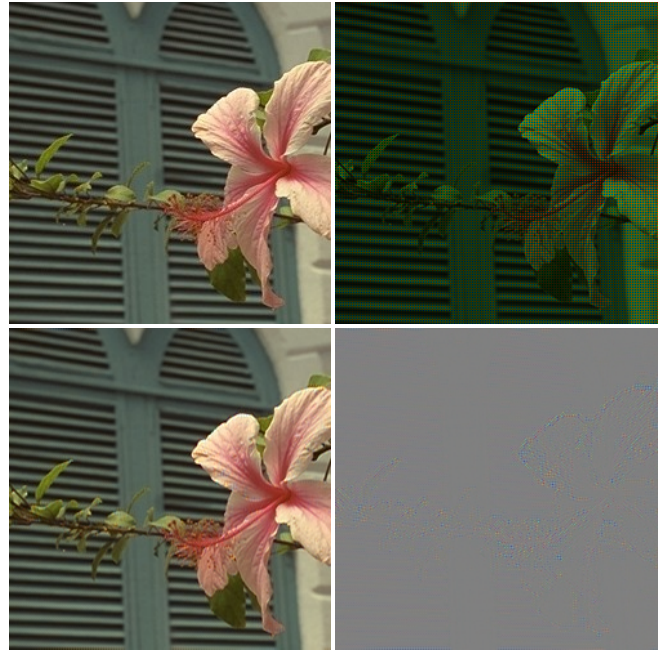

$\mathrm{NL} / \mathrm{MSH}^{1}$ with $h=\{16\}: 37.3598$

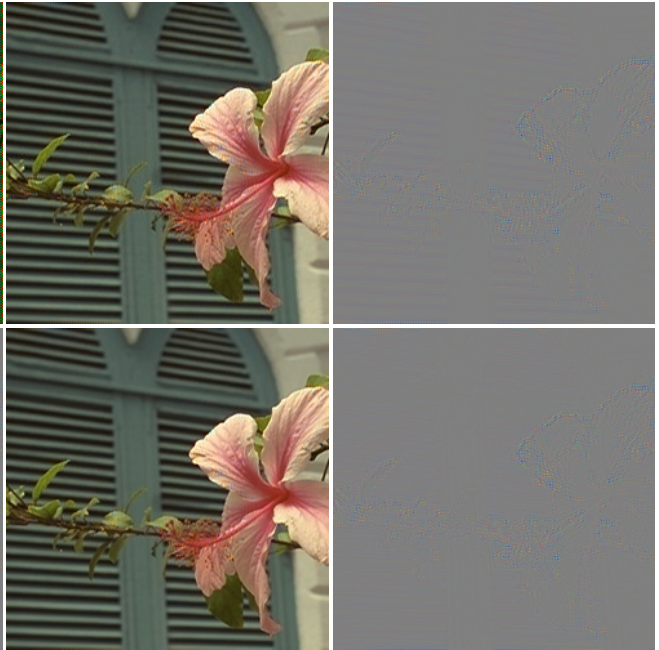

NL/MSTV with $h=\{16\}: 37.3606$

Fig. 19. Demosaiced IMAGES using NL/MS regularizers with $h=\{16\}$, and corresponding residuals with the original image. Top: original, data $f$, interpolated image with Hamilton-Adams (PSNR=36.5672) and residual. 

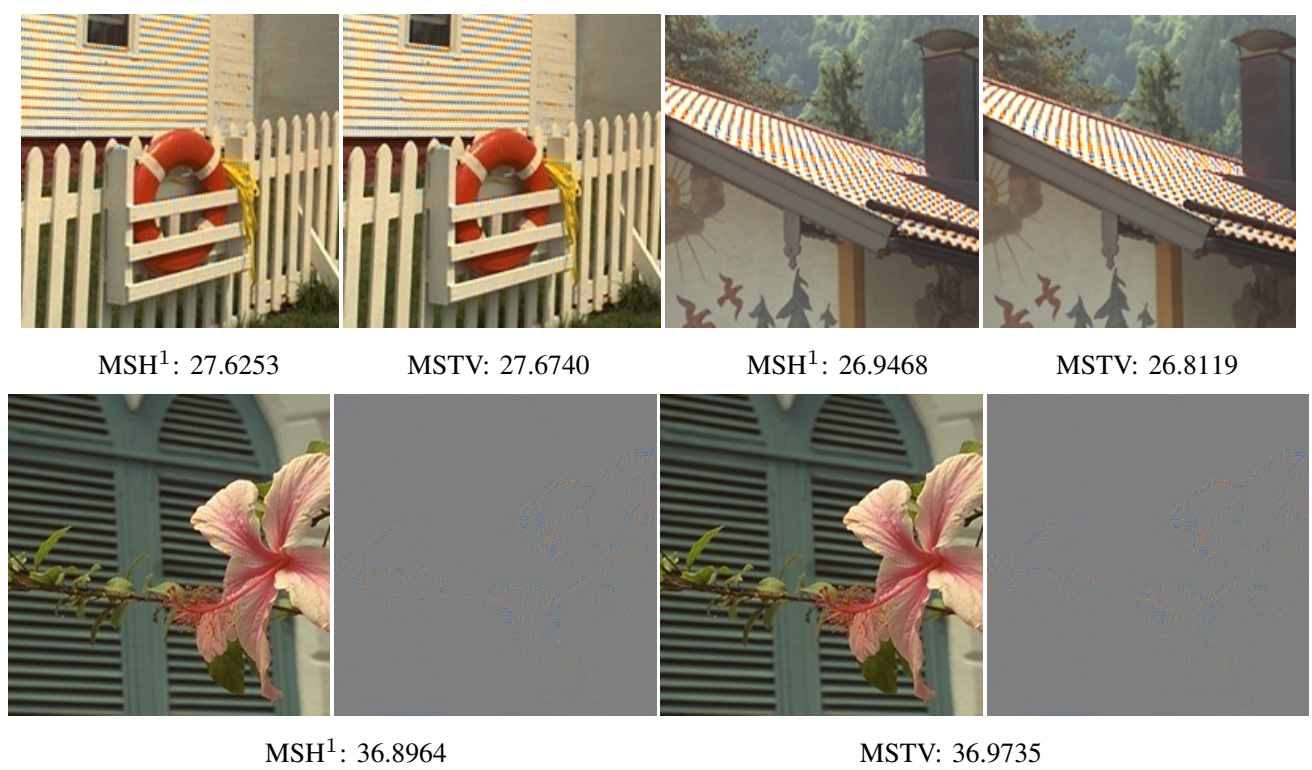

Fig. 20. Demosaiced images using local MS regularizers (for comparison).

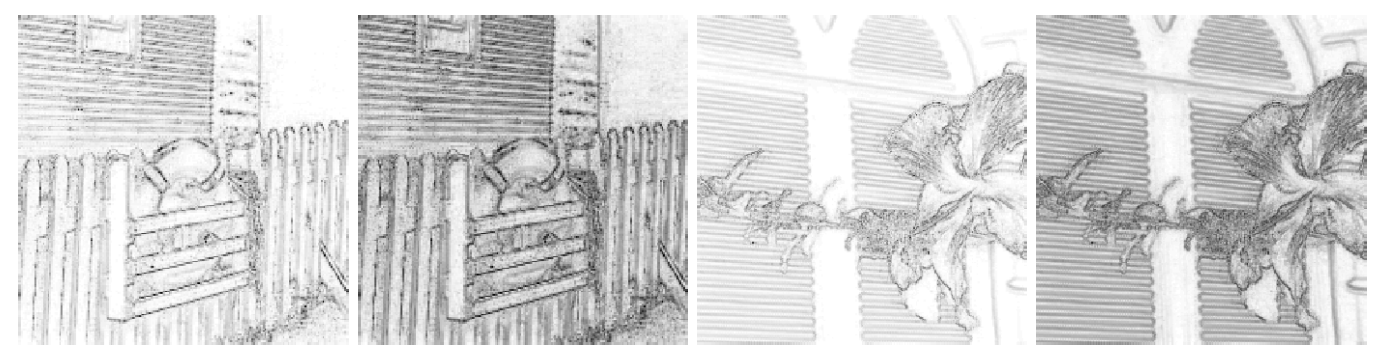

Fig. 21. Edge sets $v$ of NL/MSH ${ }^{1}$ (1st, 3rd column) and NL/MSTV (2nd, 4th column) obtained in Fig. 16 and 19 respectively. 